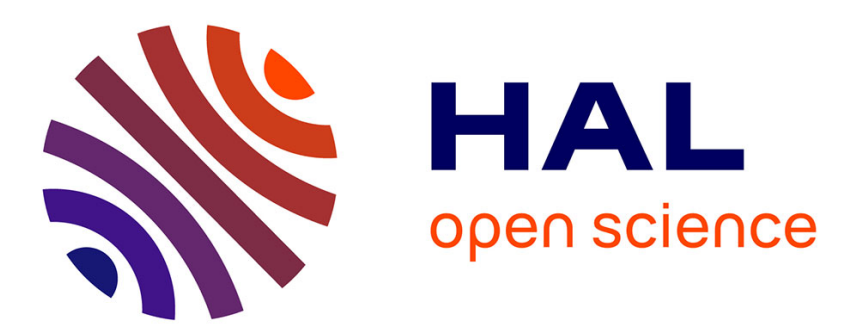

\title{
Positional dependence of particles and cells in microfluidic electrical impedance flow cytometry: origin, challenges and opportunities
}

\author{
Hugo Daguerre, Miguel Solsona, J Cottet, Michaël Gauthier, Philippe \\ Renaud, Aude Bolopion
}

\section{To cite this version:}

Hugo Daguerre, Miguel Solsona, J Cottet, Michaël Gauthier, Philippe Renaud, et al.. Positional dependence of particles and cells in microfluidic electrical impedance flow cytometry: origin, challenges and opportunities. Lab on a Chip, 2020, 20, pp.3665 - 3689. hal-02993824

\section{HAL Id: hal-02993824 \\ https://hal.science/hal-02993824}

Submitted on 7 Nov 2020

HAL is a multi-disciplinary open access archive for the deposit and dissemination of scientific research documents, whether they are published or not. The documents may come from teaching and research institutions in France or abroad, or from public or private research centers.
L'archive ouverte pluridisciplinaire HAL, est destinée au dépôt et à la diffusion de documents scientifiques de niveau recherche, publiés ou non, émanant des établissements d'enseignement et de recherche français ou étrangers, des laboratoires publics ou privés. 


\title{
Lab on a Chip
}

ARTICLE TYPE

Cite this: DOI: $00.0000 / x x x x x x x x x x$

\section{Positional Dependence of Particles and Cells in Microfluidic Electrical Impedance Flow Cytometry: Origin, Challenges and Opportunities ${ }^{\dagger}$}

\author{
Hugo Daguerre, ${ }^{* a \ddagger}$ Miguel Solsona, ${ }^{b \ddagger}$ Jonathan Cottet,,$c *$ Michaël Gauthier, ${ }^{a}$ Philippe \\ Renaud, ${ }^{b}$ and Aude Bolopion, ${ }^{a}$
}

Received Date

Accepted Date

DOI: 00.0000/xxxxxxxxxx

\begin{abstract}
Microfluidic electrical impedance flow cytometry is now a well-known and established method for single-cell analysis. Given the richness of the information provided by impedance measurements, this non-invasive and label-free approach can be used in a wide field of applications ranging from simple cell counting to disease diagnostics. One of its major limitations is the variation of the impedance signal with the position of the cell in the sensing area. Indeed, identical particles traveling along different trajectories do not result in the same data. The positional dependence can be considered as a challenge for the accuracy of microfluidic impedance cytometers. On the other hand, it has recently been regarded by several groups as an opportunity to estimate the position of particles in the microchannel and thus take a further step in the logic of integrating sensors in so-called "Lab-on-a-chip" devices. This review provides a comprehensive overview of the physical grounds of the positional dependence of impedance measurements. Then, both the developed strategies to reduce position influence in impedance-based assays and the recent reported technologies exploiting that dependence for the integration of position detection in microfluidic devices are reviewed.
\end{abstract}

\section{Introduction}

The isolation, analysis, and enumeration of some specific cell types contained in blood samples can improve the diagnosis, prognosis, and treatment monitoring of patients affected by major diseases such as cancer and cardiovascular diseases ${ }^{112}$. For instance, cells of interest can be circulating leukocytes (white blood cells) that are known to be involved in various biological processes. By becoming activated during host defense, they are essential to the immune response. In the case of cancer, tumor cells released into the bloodstream provide a sample representative of the whole tumor cell population. The frequent collection of these cells thus gives indications on tumor development and treatment effectiveness 1 .

\footnotetext{
${ }^{a}$ FEMTO-ST Institute, CNRS, Univ. Bourgogne Franche-Comté, AS2M department, 24 rue Alain Savary, F-25000 Besançon, France. Tel: +33 381402 790; Email: hugo.daguerre [at] femto-st.fr

${ }^{b}$ École Polytechnique Fédérale de Lausanne, EPFL-STI-IMT-LMIS4, Station 17, CH1015 Lausanne, Switzerland.

c Present address: Department of Mechanical Engineering, Massachusetts Institute of Technology, Cambridge, MA 02139, USA.

$\dagger$ Electronic Supplementary Information (ESI) available: Zip folder containing numerical simulation files created with Comsol Multiphysics®Version 5.5. See DOI: 00.0000/00000000.

$\ddagger$ These authors contributed equally to this work.
}

Conventional cell sorting techniques (FACS, MACS) rely on specific fluorescent or magnetic markers on the surface of target cells. Besides being usually a complex, expensive and time-consuming process, this labeling step may deteriorate the analyzed sample ${ }^{3}$. This can prevent the wide adoption of label-dependent strategies in clinical settings despite the high-throughput screening with rich content they offer. It highlights the need for label-free noninvasive sorting methods based on physical properties. In accordance with this, dielectric properties are useful to distinguish cells as they provide insight into a wide range of cell parameters such as their viability, geometry, growth or physiological state 4.6 . These properties are deduced from electrical impedance measurements that are known to enable the characterization of a wide variety of heterogeneous systems. Dielectric spectroscopy, or more generally impedance spectroscopy, is a label-free technique which has been the subject of many theoretical and technical studies over the last century 7 . An extended overview of the fundamental physical principles of the method and classical applications to systems of artificial or biological particles has been provided by Asami ${ }^{8}$. When a suspension of cells is studied, the characterization of its dielectric properties typically consists in subjecting it to an excitation voltage and measuring the resulting current over a frequency range. Electrical impedance is defined as the ratio of those two signals. It expresses the opposition of the sample to 
the electric current flow when a voltage is applied. The dielectric properties of the particles composing the disperse phase can be deduced from those of the mixture by using an appropriate set of equations modeling the system's behavior 8 . 11 .

However, such bulk assays induce an information loss by averaging the properties over the whole sample. Among a cell population of the same type and under the same physiological conditions, individual cell behavior may counterintuitively differ from one another 12 14. Because of this heterogeneity within specific cell types and as cells are the fundamental units of living organisms, investigation at the single-cell level is crucial for the understanding of the behavior of tissues, organs, and even whole living organisms 15 . It is therefore a key point for drug discovery and the development of innovative and more effective therapies 16 .

Huge advances in microengineering are supporting this growing interest for single-cell analysis by enabling the fabrication of microfluidic systems that are particularly suited to single-cell studies. They offer biologists the ability to manipulate small sample volumes and perform precise screening of individual microparticles at high throughput 17 . After some twenty years of interest in these technologies, their relevance is widely accepted and the domain is still gaining momentum 1819 .

The parallel enhancement of measurement instruments in terms of accuracy, versatility and signal processing capabilities also stimulates this research field. In particular, based on the multiplication of the measured signal with a reference waveform and the filtering of the result, so-called lock-in amplifiers are able to extract low amplitude signals from a noisy environment 20 . After decades of development, such devices have wider measurement ranges and higher repeatability, enable faster signal processing, and cover broader frequency ranges. The impedance sensor embedded in the microsystem and connected to the lock-in amplifier is typically simply composed of one or two pairs of electrodes. These electrodes are usually placed either side by side at the bottom of the microfluidic channel (coplanar configuration) or on two opposite walls facing each other (parallel/facing electrode configuration). Such sensors can be integrated in microfluidic chips by means of standard microfabrication processes, giving rise to microfluidic electrical impedance flow cytometers. Since the emergence of the latter devices, review articles regularly provide comprehensive overviews of reported systems, reflecting the dynamism of the domain 21 29. The reader is referred to those reviews and the references therein for a full insight on history and state of the art of microfluidic electrical impedance flow cytometry. A number of different microfluidic impedance cytometers have been developed until now for biomedical applications ranging from blood cell counting for disease diagnostics to microbial studies and cell phenotypic changes monitoring (e.g. 30.32 ).

However, a critical challenge for the resolution and accuracy of that impedance-based technology is the positional dependence of the measured signal. Indeed, impedance measurements depend not only on the intrinsic particle properties, but also on the particle trajectory through the sensing region. Thus, impedance signals obtained from the transit of identical particles in the microchannel can show large variations if they flow through the sensing zone along different trajectories 33 . It is an hindrance to accurate (bio)particle characterization. Given the influence of particle trajectory through the sensing area on the measured impedance, it is clear that the position has to be carefully taken into account when performing and interpreting analyses.

Numerous technical developments have been made to ensure the accuracy of impedance-based assays. Various approaches have been proposed to that end, such as controling cell position within the microfluidic channel ${ }^{35 / 38}$, compensating the positional dependence by signal processing $39 \mid 40$, and mitigating it based on specific electrode configuration or channel design $37 / 41,43$. From another perspective, the understanding and modeling of the spatial dependence of impedance measurements can be useful for the development of new integrated position sensors whose fundamental working principles are based on the aforesaid dependence 44,46 .

This study provides a comprehensive overview of the important role played by the position of particles/cells in impedance-based testing. First, the theoretical background of the impedance technique for single-cell analysis is presented, with an emphasis on its spatial dependence. Secondly, the strategies implemented in microfluidic impedance cytometers to enable accurate cell characterization despite the influence of the cell trajectory on the data obtained are reviewed. Then, recent works integrating particle/cell position detectors into microfluidic devices are described, showing that the dependence of the impedance signal on position represents an opportunity rather than an obstacle for some applications. Finally, future developments in the field of electrical impedance measurements in microfluidic devices are discussed.

\section{Theory and modeling}

Microfluidic impedance flow cytometers always rely on the same principle: individual cells flow one by one through the channel and cross sequentially a sensing zone composed of electrodes. In addition to the trajectory of the cells, the observed impedance signal depends on the electrical properties of the cells and suspending medium, on the electrode-electrolyte interface, as well as on the electrode arrangement. To predict the observed signal or interpret the obtained data in terms of cells parameters, two main strategies are commonly used: analytical, based on cell and suspension dielectric modeling as well as conformal mapping, and numerical, based on finite element mapping.

\subsection{Analytical modeling}

Impedance is defined as the ratio of the voltage applied between electrodes and the current flowing between these electrodes. Given a known applied voltage, the current measured depends on the composition of the mixture in that sensing volume, and on the nature of its interface with the electrodes. When no particle is present in this area, the properties reflected correspond only to those of the suspending medium and of the electrodeelectrolyte interface. However, as a dielectric particle or cell passes through the detection zone, it changes the properties of the medium through which the electrical signal flows. The resulting current therefore depends on the dielectric properties of both the cell and the host medium. Nevertheless, cells cannot be con- 
sidered as homogeneous. It is necessary to model the different layers that constitute them, in particular the cell membrane.

\subsubsection{Cell modeling.}

The equivalent complex permittivity of a cell is related to the properties of its different domains $11,47,50$. In the case of a cell of radius $r_{\text {ext }}$ composed of a cytoplasm surrounded by a membrane of thickness $t h_{c m}$, the "single-shell" model can be used 51 . The resulting equivalent complex permittivity $\varepsilon_{e q}^{*}$ is:

$$
\varepsilon_{e q}^{*}=\varepsilon_{c m}^{*} \frac{\left(\frac{r_{e x t}}{r_{e x t}-t h_{c m}}\right)^{3}+2\left(\frac{\varepsilon_{c p}^{*}-\varepsilon_{c m}^{*}}{\varepsilon_{c p}^{*}+2 \varepsilon_{c m}^{*}}\right)}{\left(\frac{r_{e x t}}{r_{e x t}-t h_{c m}}\right)^{3}-\left(\frac{\varepsilon_{c p}^{*}-\varepsilon_{c m}^{*}}{\varepsilon_{c p}^{*}+2 \varepsilon_{c m}^{*}}\right)},
$$

where $\varepsilon_{c m}^{*}$ and $\varepsilon_{c p}^{*}$ refer to the complex permittivities of the cell membrane and cytoplasm, which depend on their respective conductivities and permittivities, and on the electric field angular frequency $\omega$ :

$$
\varepsilon_{i}^{*}=\varepsilon_{i} \varepsilon_{0}-j \frac{\sigma_{i}}{\omega},
$$

where $\varepsilon_{i}$ is the relative permittivity, $\varepsilon_{0}$ the vacuum permittivity $\left(8.854 \times 10^{-12} \mathrm{Fm}^{-1}\right), \sigma_{i}$ the electrical conductivity $\left(\mathrm{S} \mathrm{m}^{-1}\right)$ and $\omega=2 \pi f$ with $f$ the frequency $(\mathrm{Hz})$.

The formulation of the complex permittivity for ellipsoids can be found in 52 .

\subsubsection{Cell suspension.}

For a suspension of particles or cells, the effective complex permittivity $\varepsilon_{m i x}^{*}$ depends on a parameter named the volume fraction $\varphi$ according to the Maxwell Mixture Theory (MMT) 53|54 whose validity domain is restricted to a small cell in an homogeneous electric field. It is given by the Maxwell-Garnett mixing equation if the volume fraction $\varphi$ is smaller than 0.1 :

$$
\frac{\varepsilon_{m i x}^{*}-\varepsilon_{m}^{*}}{\varepsilon_{m i x}^{*}+2 \varepsilon_{m}^{*}}=\varphi \frac{\varepsilon_{p}^{*}-\varepsilon_{m}^{*}}{\varepsilon_{p}^{*}+2 \varepsilon_{m}^{*}},
$$

where index $p$ and $m$ refer to the particle and the medium respectively. This formulation is equivalent to the direct formulation 55 .

$$
\varepsilon_{m i x}^{*}=\varepsilon_{m}^{*}\left(1+3 \varphi \frac{\frac{\varepsilon_{p}^{*}-\varepsilon_{m}^{*}}{\varepsilon_{p}^{*}+2 \varepsilon_{m}^{*}}}{1-\varphi \frac{\varepsilon_{p}^{*}-\varepsilon_{m}^{*}}{\varepsilon_{p}^{*}+2 \varepsilon_{m}^{*}}}\right) .
$$

Such modeling can be used to analyze experimental data and determine the value of the different (bio)particle parameters through a fitting algorithm 56 .

If the volume fraction is greater than 0.1 , the Hanai equation 9 , theoretically valid up to $\varphi<0.8^{\frac{57 / 58}{58}}$ should be implemented:

$$
\left(\frac{\varepsilon_{m i x}^{*}-\varepsilon_{p}^{*}}{\varepsilon_{m}^{*}-\varepsilon_{p}^{*}}\right)\left(\frac{\varepsilon_{m}^{*}}{\varepsilon_{p}^{*}}\right)^{1 / 3}=1-\varphi .
$$

Unfortunately, there is no direct expression for the calculation of $\varepsilon_{\text {mix }}^{*}$ from the Hanai formulation. This value can therefore be obtained either by solving the cubic equation or by numerical integration with the difference equation of Hanai's equation. The latter is implemented in the software MyDEP ${ }^{59}$ with the full methodology explained in the supplementary material of that article and in 1060 . For higher volume fractions, the Hanai formulation has proven to be more accurate than the Maxwell-Garnett formulation 61 . However, variation of the vertical position of the particle has shown that both models exhibit significant errors when the particle is in close vicinity to the electrodes.

It is important to notice that the calculation of $\varepsilon_{m i x}^{*}$ does not depend on the position of particles/cells as the derivation assumes an homogeneous electric field.

\subsubsection{Volume fraction.}

The volume fraction $\varphi$ is normally defined for a parallel electrode geometry with uniform electric field distribution by the volume occupied by cells over the entire sensing volume 21 .

However, in microsystems, as electrodes are not of infinite size and the volume of liquid between them is not completely confined this formulation should be corrected. Such correction, based on conformal mapping was used by Morgan et al. 21 for facing electrodes with a centered particle. The formulation used by the authors therefore does not take into account the variation of position of the particle. It is only suited to systems where the particles are located at the middle point between electrodes.

Similarly, regarding coplanar electrodes, no analytical formula with large validity domain has been found in the literature to calculate the corresponding volume fraction. Alternatively, Liu et al. 62 proposed a graphical conformal mapping method to account for the non-uniformity of the electric field and the different positions of the particles. Such method relies on the graphical determination of the cell size in a grid composed of electric field lines and equipotential lines using image processing techniques as presented in Fig. 11. The calculation of the equipotential lines requires the use of Finite Element Analysis (FEA).

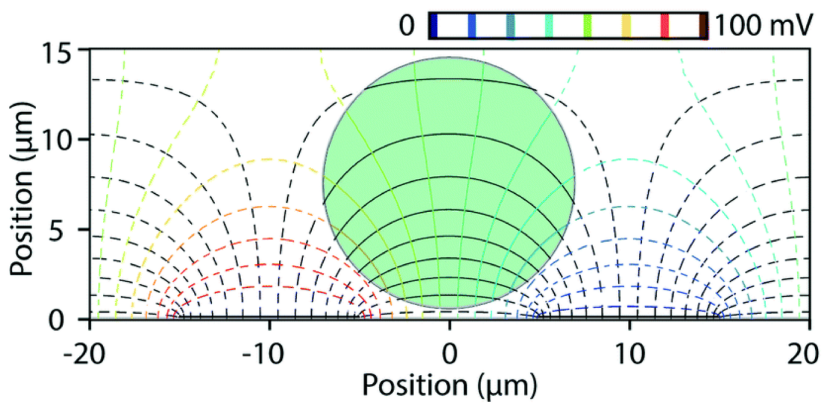

Fig. 1 Example of graphical conformal mapping. Adapted from Liu et al. 62 with permission from The Royal Society of Chemistry, copyright 2017.

\subsubsection{Impedance in microsystems.}

The complex impedance of the cell suspension $Z_{m i x}^{*}$ is linked to its complex effective permittivity $\varepsilon_{\text {mix }}^{*}$ by:

$$
Z_{m i x}^{*}=\frac{1}{j \omega \varepsilon_{m i x}^{*} G},
$$

where $G$ is a constant only depending on the geometry of the sensing zone and used as correction factor of the fringing field in that zone due to the edge effect $21 / 22 / 33$. For an ideal facing 
electrodes system this constant is equal to the electrode surface divided by the inter-electrode distance.

To account for the non-uniformity of the electric field distribution, Gawad et al. .33 proposed a methodology based on conformal mapping to calculate $G$ for facing electrodes. Jacobs et al $\underline{63}$ introduced a methodology for a coplanar electrode configuration with infinite height which was later extended by Linderholm et al. $\frac{64}{6}$ for coplanar geometries with finite channel height.

\subsubsection{Equivalent circuit modeling.}

A commonly used technique to account for the cell-electrode interaction is the developement of an equivalent circuit model called the Foster-Schwan model ${ }^{65}$. In this approach, the behavior of each material is modeled by both a resitor element in parallel with a capacitive element. The resistive element accounts for the movement of free charges (corresponding to the electrical conductivity) while the capacitive element symbolizes the fieldinduced perturbation of bound charges (as represented by the permittivity). The modeling of such behavior, mathematically visible in Equation 2, is frequency dependent as the conductive behavior will be mostly visible at lower frequencies and the charging mechanism at higher frequencies 66 .

The models used to represent the different effects visible in the impedance spectra evolved from the simple resistor and capacitor in parallel to depict the behavior of a homogeneous particle 67 to the addition of the properties of the membrane represented by both a capacitor and resistor in series with the previous parallel model ${ }^{21 \mid 68}$. Such modeling reflects the frequency dependence of the impedance signal: at low frequencies, the cell membrane behaves as an insulator with a capacitive effect and the impedance signal is mostly sensitive to the volume of the particles and the electrode-electrolyte interface. At higher frequencies, the impedance of the cell membrane capacitor decreases and the interior of the cell becomes accessible to the electric field.

In addition, the electrode-electrolyte interface behaves like a capacitance called the electrochemical double-layer capacitance which represents the charging of the electrode surface in contact with the solution and depends on the material and surface area of the electrode. An improved method commonly adopted to model the electrochemical double-layer is to use a constant phase element (CPE) 69. 71 .

\subsubsection{Conclusion on analytical modeling.}

Most of the analytical modeling, despite providing a first insight into the impedance variation, are very limited when particle position changes. In particular, the high non-homogeneity of the electric field close to the electrodes cannot be neglected. In both standard electrode configurations (i.e. parallel and coplanar), the particle properties deduced by the application of the MMT are only valid for a centered particle of small size compared to the inhomogeneity of the electric field in the sensing zone (i.e. where the electric field is quasi homogeneous and the geometric factor is known).

To overcome such limitation and the usage of mathematical tools such as conformal mapping which are limited to 2D symmetrical geometries, numerical modeling is often exploited.

\subsection{Numerical modeling}

Nowadays, the most common method for modeling the electrical response of a cell in suspension is numerical simulation by Finite Element Method (FEM). Since electrodes are integrated into the channel walls and are of comparable size as the analyzed cells, the high non-homogeneity of the electric field cannot be neglected. FEM is particularly suited for solving such physical problems with a complicated field determined by partial differential equations. It is a tool widely used in the field of bio-MEMS in device design to predict the impedance change induced by a cell of given parameters (size, membrane permittivity, etc.) over a specific range of excitation frequencies 3372 . The use of most commercial software is quite intuitive and the whole problem solving process is divided into a few steps ${ }^{73}$ :

\section{Defining of the geometry}

It consists in constructing the channel and the electrodes similarly to what would be done in Computer-Aided Design (CAD) software. However, modeling the cell is not as simple as this because the thickness of the membrane is very small compared to cell radius. Such a large geometric ratio could be a problem for the meshing and the accuracy of the solution. This is the reason why the cell is modeled as an homogeneous solid sphere whose electrical properties are calculated using an analytical model of the cell according to its different parts (membrane, cytoplasm...) as presented previously. Instead of a geometrical entity, Cottet et al. 37 proposed to define it as a change in material properties. Several cell models have been reported in the literature so far, allowing to treat different types of cells. The relationship between cell equivalent properties and the properties of the different domains composing the cell is given in the literature for different cell types $8[11,47,50$.

\section{Setting of the boundary conditions}

Two types of boundary conditions are set. Dirichlet boundary conditions are applied on the electrode surfaces: typically, $\varphi=V$ (excitation voltage) and $\varphi=0$ (ground), while Neumann boundary conditions $\frac{\partial \varphi}{\partial n}=0$ are applied on all other surfaces. The electric field is assumed to be completely contained in the modeled channel, which is true if the channel is long enough compared to the electrode width (i.e. most of the energy is contained in the channel).

\section{Meshing and setting of the solver parameters}

An appropriate mesh should be chosen for the problem studied. The size of the mesh will be paramount in the accuracy of the provided solution. The entire domain is divided into smaller elements where the Laplace equation is solved. A very fine mesh will be more accurate than a coarser mesh but will require a lot of computational power. To reduce the size of the meshed volume, symmetries, shall they be present in the studied geometry, should be indicated. 


\section{Solving and post processing}

The FEA software solves Laplace's equation in both domains (cell represented by the homogeneous sphere and the fluidfilled channel) taking into account the previously set boundary conditions. The created study is usually solved in the frequency domain at a defined frequency. As the voltage between electrodes is fixed, the current corresponds to the integral of the current over the excitation or ground electrode.

Numerical simulations offer the ability to simulate any particle position and are not limited to particle centered between the electrodes. Any position of the particle can therefore be simulated and the results obtained often are in good agreement with the experimental results. They also offer the possibility to simulate the dynamic signal measured by the sensor when a particle passes through. Such simulations require to manually move the particle in the channel 37 or to use a moving mesh which is very demanding in term of computing power and is normally reserved for deformable particles such as Red Blood Cells (RBCs) 74/75. In addition, the electrical double layer can also be directly modeled in most software.

\subsection{FEM comparison of facing and coplanar electrodes}

In order to be able to compare the two most widely spread configurations of electrodes used for impedance detection in microsystems, the facing and the coplanar electrode designs, FEM simulations were performed using the AC/DC Module of Comsol Multiphysics 5.5.

The different simulations were carried out to study the impedance variation due to a polystyrene (PS) bead of radius $4 \mu \mathrm{m}$ positioned at the center of the detection volume $(x=0 \mu \mathrm{m}$, $y=0 \mu m, z=h / 2=10 \mu m$ ). The bead and liquid electrical conductivities and relative permittivities values were set to respectively $5 \times 10^{-6} \mathrm{~S} / \mathrm{m}$ (respectively $1.6 \mathrm{~S} / \mathrm{m}$ ) and 2.5 (respectively 80 ). The microchannel was set to be $20 \mu \mathrm{m}$ wide, $20 \mu \mathrm{m}$ high and $180 \mu \mathrm{m}$ and $140 \mu \mathrm{m}$ long for the coplanar and facing electrodes respectively. Electrodes were $20 \mu \mathrm{m}$ wide, $20 \mu \mathrm{m}$ long and separated by $20 \mu \mathrm{m}$ for the coplanar configuration. The simulations used a swept mesh with an element size of $1 \mu \mathrm{m}$ and a solver tolerance of $1 \mathrm{e}-7$.

As presented in Cottet et al. $\frac{37}{3}$, a spherical particle was defined as a change in material properties instead of a geometrical entity. The particle was then moved along the microchannel by displacing its center using a parametric sweep. For both configurations an AC signal of amplitude $100 m V_{p \text { (peak) }}$ at $500 \mathrm{kHz}$ was applied on the excitation electrode, set to a terminal, while the receiving electrode was set to ground. The results of the simulations are presented in Fig. 2

In order to help the reader to reproduce and adapt the presented simulations, the full methodology is provided in the supplementary material $^{\dagger}$ of this article as well as two Comsol simulation files (version 5.5) for the facing and the coplanar electrode designs. The specifications of the simulation computer are also detailed together with a discussion of the different meshing possibilities and their computational time.
The maximum impedance variation for a particle of radius $4 \mu \mathrm{m}$ centered in the channel cross-section is of $3.27 \%$ and $2.61 \%$ for the coplanar and facing electrode configuration respectively. It is obtained when the particle is located exactly in the center between the two electrodes.

For both configurations the lateral and vertical sensitivities are presented in Fig. 3. It turns out that variations of particle lateral position influence impedance variation up to $2.2 \%$ in the facing design and $2.9 \%$ in the coplanar design, both with respect to the impedance variation induced by a centered particle. Considering the lowest/highest z positions of the particle $(z=5 \mu \mathrm{m}$ and $z=$ $15 \mu \mathrm{m}$ ), the variation of the impedance variation is of $4.2 \%$ in the facing design and $+46.2 \% /-21.4 \%$ in the coplanar design, both compared to a z-centered particle $(z=h / 2=10 \mu \mathrm{m})$.

It is worth mentioning that the impedance variation due to particles flowing close to the electrodes in coplanar designs exhibits a $\mathrm{M}$ shape 37 . As presented in Fig. 4, this $\mathrm{M}$ shape is enhanced by a decrease of the inter-electrode distance and/or by a lower altitude of the particles.

\subsection{Conclusion on theory and modeling}

Despite their ease-of-use and their ability to solve complex problems in most cases without resorting to simplifying hypothesis, FEM solutions suffer from certain disadvantages compared to analytical models. Since the solution accuracy is closely related to the mesh size, a large number of refined elements is often required. This significantly increases the computational time and cost of the computational capacity needed to obtain accurate results $76 / 77$. Confidence in the obtained results depends on the tolerance set for the solving as well as the validation of the mesh used through a mesh convergence study 37 . Error calculation can also be used for adaptive mesh refinement in which the error estimate is used to refine the areas in the volume where the error is the largest $61 / 78$.

Both analytical modeling and numerical simulations are used for the design of microfluidic chips. While analytical modeling can only provide an estimate of the impedance value at a specific position, numerical simulations offer the possibility to analyze any geometry with particles in any position as long as the mesh and the computing power are sufficient. Real-time analysis requires the construction of template library which can be built with numerical simulations and template recognition 62 .

The impedance variation due to cell position in a given sensing region should always be compared to the natural dispersion of cell properties within the analyzed population. Several examples can be found in the literature together with a discussion of the electrical impedance characterization such as for RBCs ${ }^{79} 80$, T-lymphocytes, Monocytes and Neutrophils ${ }^{81}$, Yeast cells ${ }^{82 / 83}$. If the measured signal in impedance-based single-cell analysis depends significantly on cell position, the accurate characterization of the cell from the impedance signal requires either to avoid signal variations due to trajectory changes, or to compensate them. The next section of the paper is dedicated to the different strategies that have been developed for that purpose when (bio)particles go though impedance microsensors. 


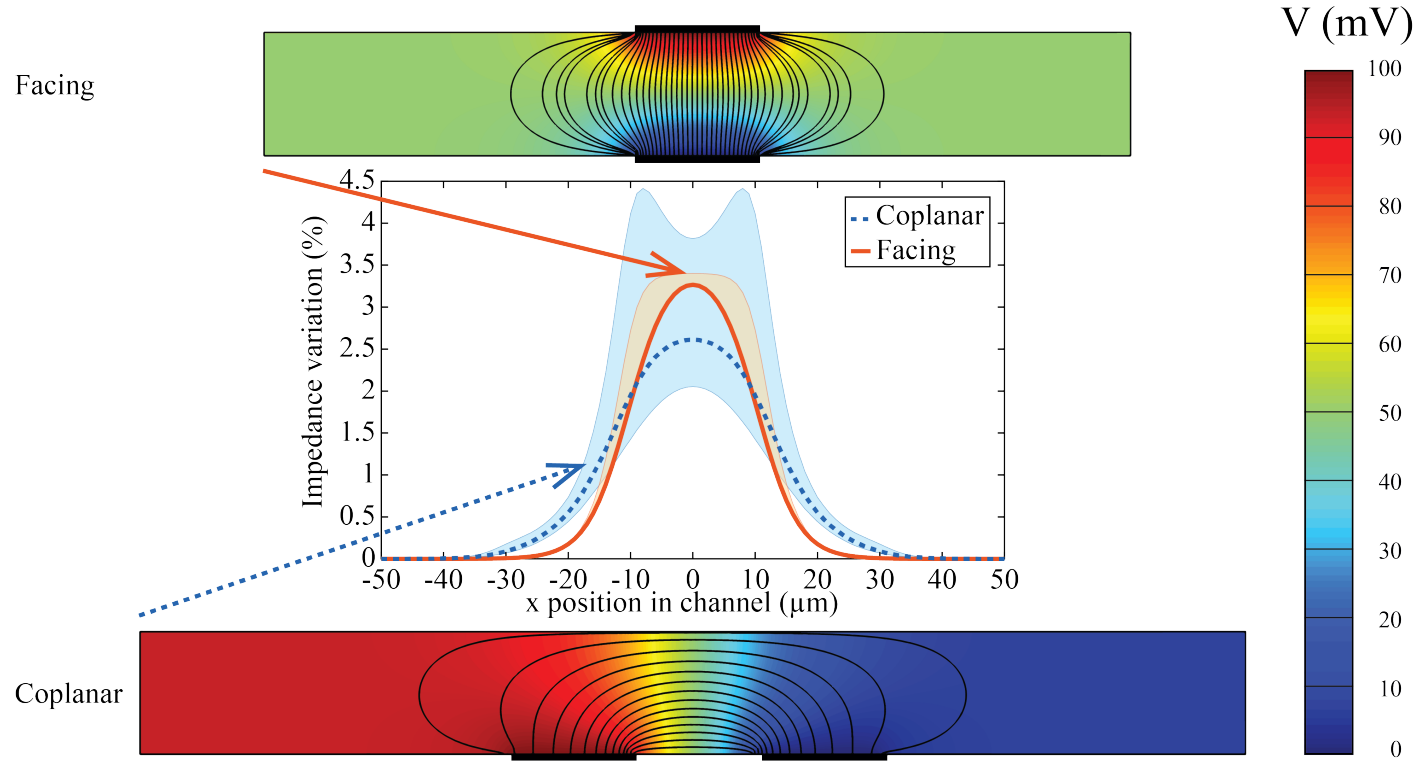

Fig. 2 Map of the electric potential distribution for the facing (top) and coplanar (bottom) electrode configurations with the electric field lines in black. (Center) Impedance variation due to the transit of a centered particle in the microchannel $(x=0 \mu \mathrm{m}, y=0 \mu \mathrm{m}, z=h / 2=10 \mu \mathrm{m})$ for both configurations (thick lines) and variation between the lowest and highest altitudes for both designs (lighter colour zone).
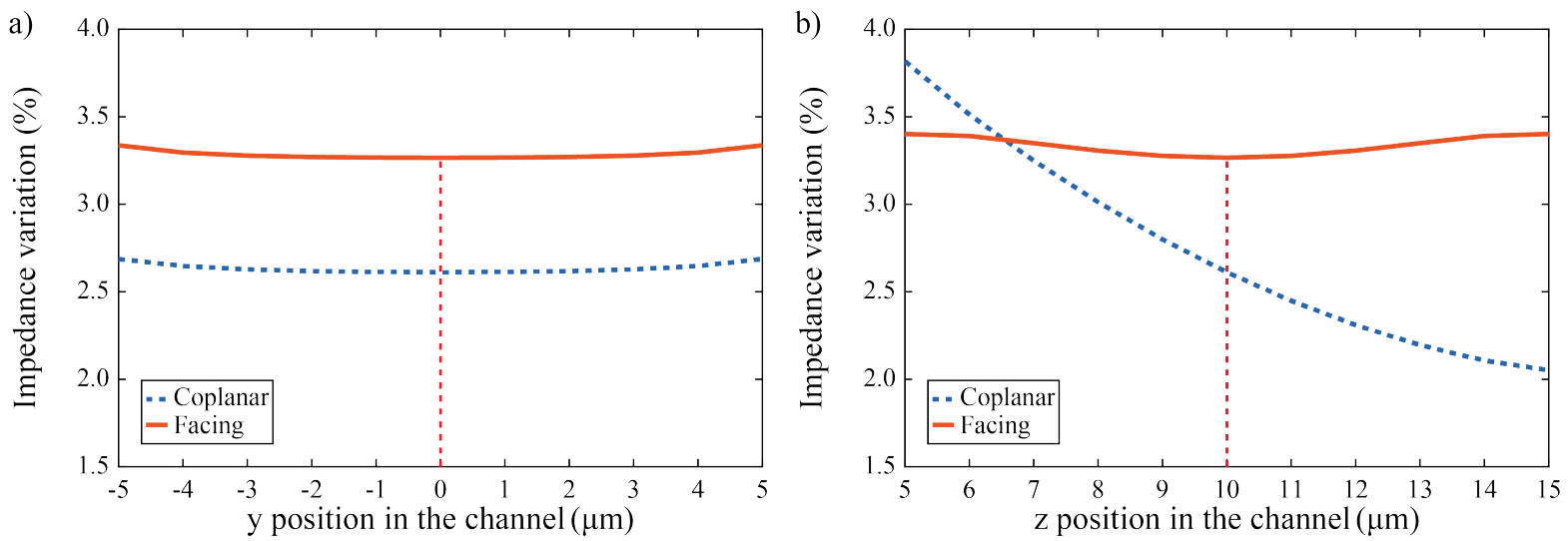

Fig. 3 Simulation of the current variation due to an $8 \mu \mathrm{m}$ diameter polystyrene bead for the facing and the coplanar designs. Evolution with a) the $y$ position in the microchannel (located at $(x=0 \mu \mathrm{m}, z=h / 2=10 \mu \mathrm{m})$ and with b) the $z$ position in the microchannel (located at $(x=0 \mu \mathrm{m}, y=0 \mu \mathrm{m})$. The red vertical dotted line represents the position of the particle in the center $(x=0 \mu \mathrm{m}, y=0 \mu \mathrm{m}, z=h / 2=10 \mu \mathrm{m})$.
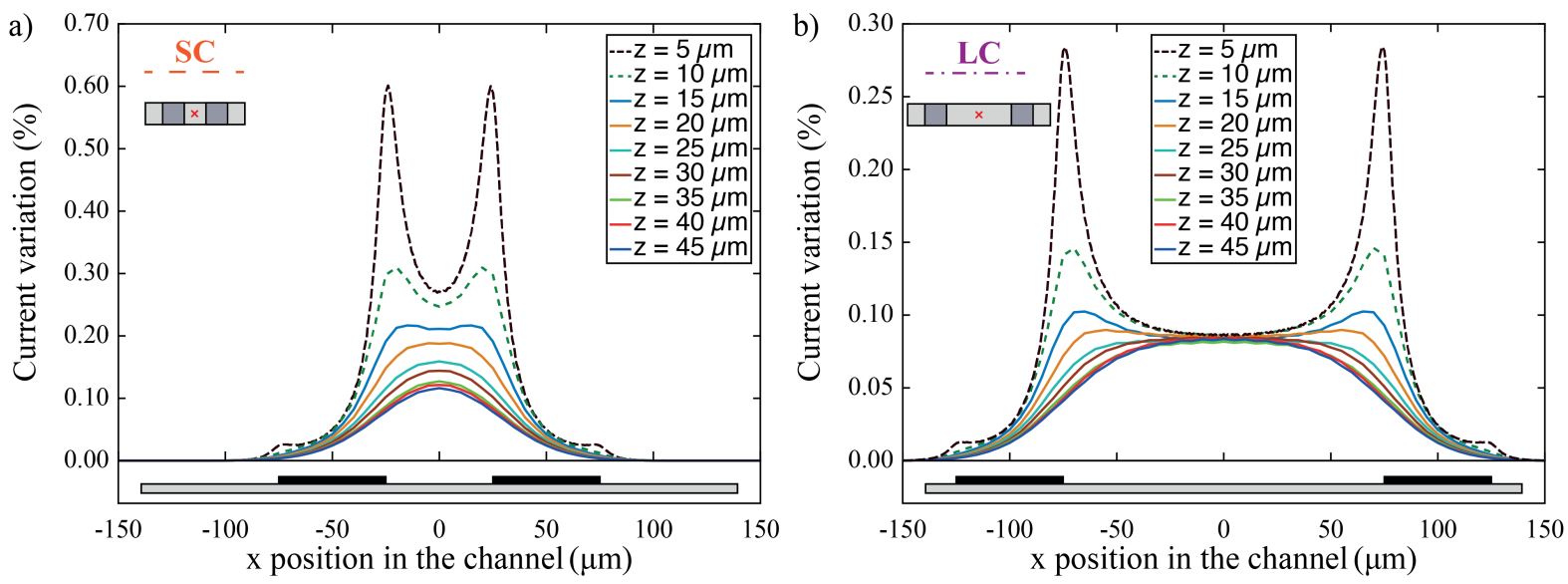

Fig. 4 Simulation of the current variation due to an $8 \mu \mathrm{m}$ diameter polystyrene bead. Evolution with the $\mathrm{x}$ position in the microchannel (located at $y=0 \mu \mathrm{m}$ ) at different heights for conventional coplanar designs with an inter-electrode distance of $50 \mu \mathrm{m}$ (SC) and $150 \mu \mathrm{m}$ (LC). Electrode positions (in black) are indicated under each graph. Adapted by permission from Cottet et al. $\frac{37}{3}$, Springer Nature, copyright 2019. 


\section{Methods to overcome the positional de- pendence in cell/particle analysis}

As illustrated by Fig. 2 and 3 , the variation of the impedance signal as a function of cell trajectory is highly dependent on the design of the sensing zone. In some cases, depending on the heterogeneity of the analyzed sample, position variations can be the cause of an unexpected spread in cells/particles extracted features $33.35 \mid 84$.

Great efforts have been devoted to overcome or mitigate this limitation. Even if a narrow channel would obviate the need for focusing, a smaller channel section would increase the risk of clogging as well as the pressure drop. Although microfluidic devices with constriction channels have been developed ${ }^{85 / 86}$, their ability to handle a large range of different sized particles remains limited compared to classical microfluidic devices. To cope with the positional dependence without the associated risk of clogging the channel, other methods have been developed. They can be divided into two categories: particle focusing methods and focusing-free methods. The first category relies on a consistent control of cell trajectory within the microfluidic channel to ensure repeatable accurate impedance-based measurements. Alternatively, the second category is based either on the compensation of position variations by signal processing, or on specific electrode designs enabling the reduction of the dependence of the impedance signal on particle position.

\subsection{Particle focusing}

Even if it sometimes increases the level of complexity of the instrument, adding a system to control particle trajectory through the channel appears to be the most obvious solution to overcome challenges induced by the positional dependence. Numerous particle focusing techniques have been developed and studied so far. The following sections will particularly focus on presenting the main ones and on giving examples of recently reported microfluidic impedance cytometers integrating them. For a deeper analysis of each particle focusing technique beyond its use in the field of impedance flow cytometry, the reader is referred to dedicated articles 27338 .

\subsubsection{Hydrodynamic sheath flow focusing.}

Besides their use as particle and cell sorting actuation means ${ }^{87}$, hydrodynamic flow focusing methods have been used for a long time together with impedance-based particle counting and/or characterization in microfluidic devices 88 94. Such methods enable the control of the particle path through the channel. This is typically done by using one or more secondary particle-free fluid streams to compress the particle suspension flow and thus focus particles into a single streamline (Fig. 5). In other words, it consists of sheath fluids flowing at a higher pressure enabling the pinching of the core fluid containing the particles ${ }^{95}$. The focusing is therefore realized without resorting to the manipulation of the particles themselves but by steering the whole particle suspension flow. For this reason, its potential to focus the sample into a narrow stream is governed by the ratio of the sheath fluid flow rate to the flow rate of the sample. This method has the ad-

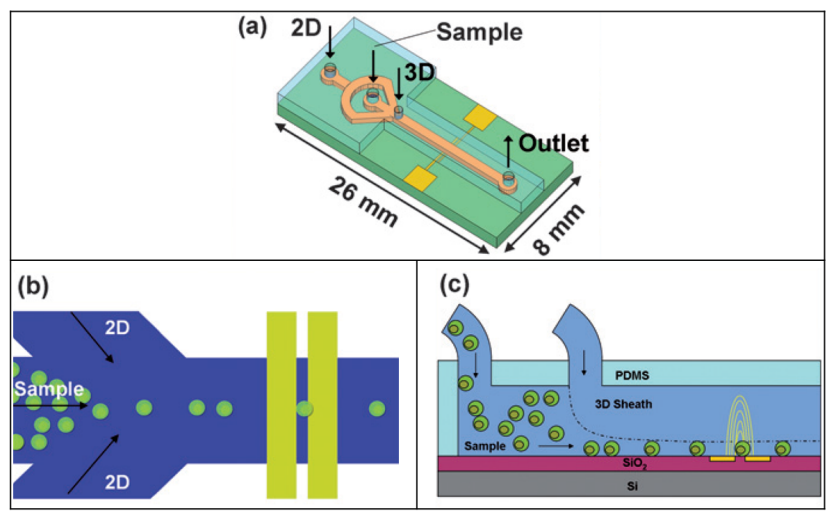

Fig. 5 Illustration of 2D and 3D hydrodynamic sheath flow focusing technique. Adapted from Watkins et al. ${ }^{96}$ with permission from The Royal Society of Chemistry, copyright 2009.

vantage of being tunable as both the location and the dimension of the focused particle stream can be adjusted using the flow rates of each sheath fluid as control parameters.

The focusing performed is said to be either two-dimensional (2D) or three-dimensional (3D) according to the degree of freedom given to the sample flow. 2D hydrodynamic focusing refers to systems that generate a sheet of particles i.e. a planar interface between the sheath and sample flows, while 3D focusing systems focus the particles into a beam, giving rise to a three-dimensional interface as presented in Fig. 5 .

2D sheath flow focusing usually enables the control over the horizontal position of the sample stream, while particle position over channel height is free. The lateral focusing plane is often chosen to be the center plane of the microchannel. It is typically done by using a Y (or T)-shaped microfluidic channel and two equal sheath flows coming from the side inlets constraining laterally the sample flow coming from the central inlet. A two-inlets microfluidic channel can be used, reducing the complexity of use of the device when the application does not require to change the focusing plane over time (Fig.5 5 ,b).

The device presented by Bernabini et al. .97 illustrates the use of $2 \mathrm{D}$ hydrodynamic focusing in the context of microfluidic impedance cytometry (Fig. 6). The electrodes composing the impedance sensor were placed facing each other instead of side by side, giving a more uniform electric field ${ }^{76}$. Moreover, the insulating sheath fluid (chosen to be oil in this work) not only focuses the sample stream within the channel, but also reduces the sensing volume. It can be considered as channel movable vir-

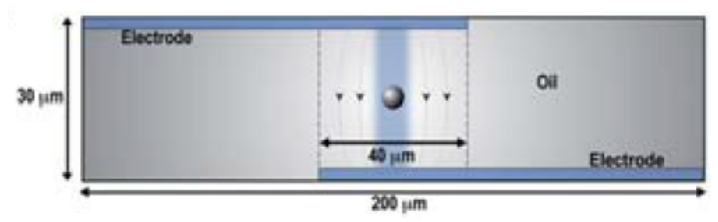

Fig. 6 Cross-section view of the microchannel showing the lateral focusing of the sample stream using an insulating sheath fluid. Adapted from Bernabini et al. $\frac{97}{}$ with permission from The Royal Society of Chemistry, copyright 2011. 
tual walls 98 . The current density is confined to the Phosphate Buffered Saline (PBS) solution, increasing both the uniformity of the electric field and the sensitivity of the sensor. While maintaining relatively large channel dimensions (200 $\mu \mathrm{m}$ width and $30 \mu \mathrm{m}$ height), hydrodynamic focusing in two dimensions allows control of the sensing volume (down to around $10 \mu \mathrm{m}$ wide in the described experiment). This volume can be varied within an experiment, or from an experiment to another, enabling the analysis of a wide range of particles. The impedance-based discrimination between similarly sized particles has been presented 97: $1 \mu \mathrm{m}$ and $2 \mu \mathrm{m}$ diameter beads, and $E$. coli bacteria and $2 \mu \mathrm{m}$ beads. Bead size dispersion calculated from impedance measurements turned out to be in good agreement with the manufacturer quoted values. Regarding the $E$. coli, the estimated coefficient of variation in size was $6 \%$. Such a tight distribution was expected as the bacteria were in stationary phase.

Even if the measured impedance is made less sensitive to particle height as done by Bernabini et al. 97 , the use of $2 \mathrm{D}$ focusing in microfluidic flow cytometers suffers from some problems such as the probability of coincident detection due to the simultaneous passage of two or more particles in the detection region. As the width of the sample flow is adapted to suit particle diameter, two particles cannot travel side by side. Nevertheless, nothing prevents them from flowing one above the other. This problem is not encountered in 3D focusing, as particles are focused in both the horizontal and the vertical directions 96|99|100.

\subsubsection{Inertial focusing.}

Particle focusing can also be achieved by taking advantage of fluidic inertial effects. As a "passive" technique, it enables particles and cells manipulation in microchannels without the need for sheath flows or any externally applied field. Contrary to the widespread idea that inertia can be neglected in microfluidics, various effects based on the fluid inertia have been shown to be useful for separation applications ${ }^{105}$ and (bio)particle focusing $103 / 106,108$.

The fundamental fluid dynamic principles on which that focusing method is based have been comprehensively explained in dedicated reviews $101 / 102 \mid 109$. Fluid flows are generally described by several dimensionless parameters among which the Reynolds number $R e$ gives informations about flow regime (typically creeping, laminar, transition or turbulent). It represents the ratio of the inertial forces to the viscous effects. For a rectangular channel of height $h$ and width $w$ (in meter) giving a channel hydraulic diameter $D_{h}=\frac{2 w h}{w+h}$, it is defined as:

$$
R e=\frac{\rho U D_{h}}{\mu},
$$

where $U$ is the maximum fluid velocity (m.s ${ }^{-1}$ ), and $\mu$ and $\rho$ are fluid dynamic viscosity (Pa.s) and density $\left(\mathrm{kg} . \mathrm{m}^{-3}\right)$, respectively. Unlike traditional microfluidic technologies using Stokes flow (also called creeping flow), where fluid inertia is negligible and flow occurs at very low Reynolds number (i.e. $R e \ll 1$ ), inertial microfluidics works in the middle regime where both inertia and viscosity are significant but the flow remains laminar (i.e. between Stokes and turbulent regimes $\sim 1<R e<\sim 2000$ ). In such a regime, particles flowing in a microchannel experience nonnegligible hydrodynamic inertial lift forces, thus undergo crossstreamline migration to a set of equilibrium positions depending on the channel geometry (Fig. 7). In addition to the Reynolds number, which describes the unperturbed flow, another dimensionless number can be defined to describe the flow of particles in closed channel systems, namely the particle Reynolds number $\left(R e_{p}\right)$. It is based on parameters describing both the flowing particle and the channel. For a rectangular channel, it is defined as 103 .

$$
\operatorname{Re}_{p}=\operatorname{Re}\left(\frac{a}{D_{h}}\right)^{2}=\frac{\rho U a^{2}}{\mu D_{h}},
$$

where $a$ is particle diameter (in meter). It turns out that the greater the particle Reynolds number is, the more inertial lift forces dominate particle behavior, leading to a faster and clearer migration across streamlines. $R e_{p} \ll 1$ is typical for particle flow in microscale channels dominated by viscous interactions. As $R e_{p}$ increases and becomes closer to 1 , inertial focusing effects become significant. In particular, previous studies reported critical Reynolds number for different channel geometries and the general conditions for achieving clear inertial focusing 110 . $a / D_{h}>0.07$ and $R e_{p}>1$. In concrete terms, it means that, being related to fluid inertia, these phenomena are only relevant at high flow rates. These conditions also highlight the influence of particle size in inertial focusing. At a given $R e$, large particles migrate faster than small particles and the focusing position is not the same.

As shown in Fig. 7, the number of equilibrium positions is large in tubular straight channels, while it is unique in asymmetrically curved channels and respectively four and two in square and rectangular straight microchannels. More precisely, these equilibrium positions are located in the middle of each wall in square channels and in the middle of the longer walls in rectangular channels. Zhou and Papautsky 102 showed that particle cross-stream motion in rectangular channels occurs in two stages: fast migration toward the longer walls followed by a slow migration parallel to channel walls into wall-centered equilibrium positions.

The non-uniqueness of the focusing position in such simple designs can pose difficulties in impedance flow cytometry applications, such as coincident detection or non-repeatable measurements if the electric field in the sensing zone differs from one equilibrium path to another. This is the reason why some studies have explored the possibility to go beyond classical cross-sectional geometries in order to achieve the focusing of microparticles to a single stream ${ }^{104} 111$ as presented in Fig. 8 However, these fundamental works also show that inertial focusing of heterogeneous particles samples remains complex because of focusing variation as a function of particle size. It can be an issue in the context of impedance cytometry, according to the degree of heterogeneity within the analyzed cell population ${ }^{31 / 112}$.

And yet, inertial focusing has already been used in microfluidic impedance flow cytometers 106 . For instance, in 2019 in the work of Raillon et al. for label-free isolation and enumeration of Circulating Tumor Cells (CTCs) from blood samples 108 . By means of a rectangular microchannel, the sample was focused 

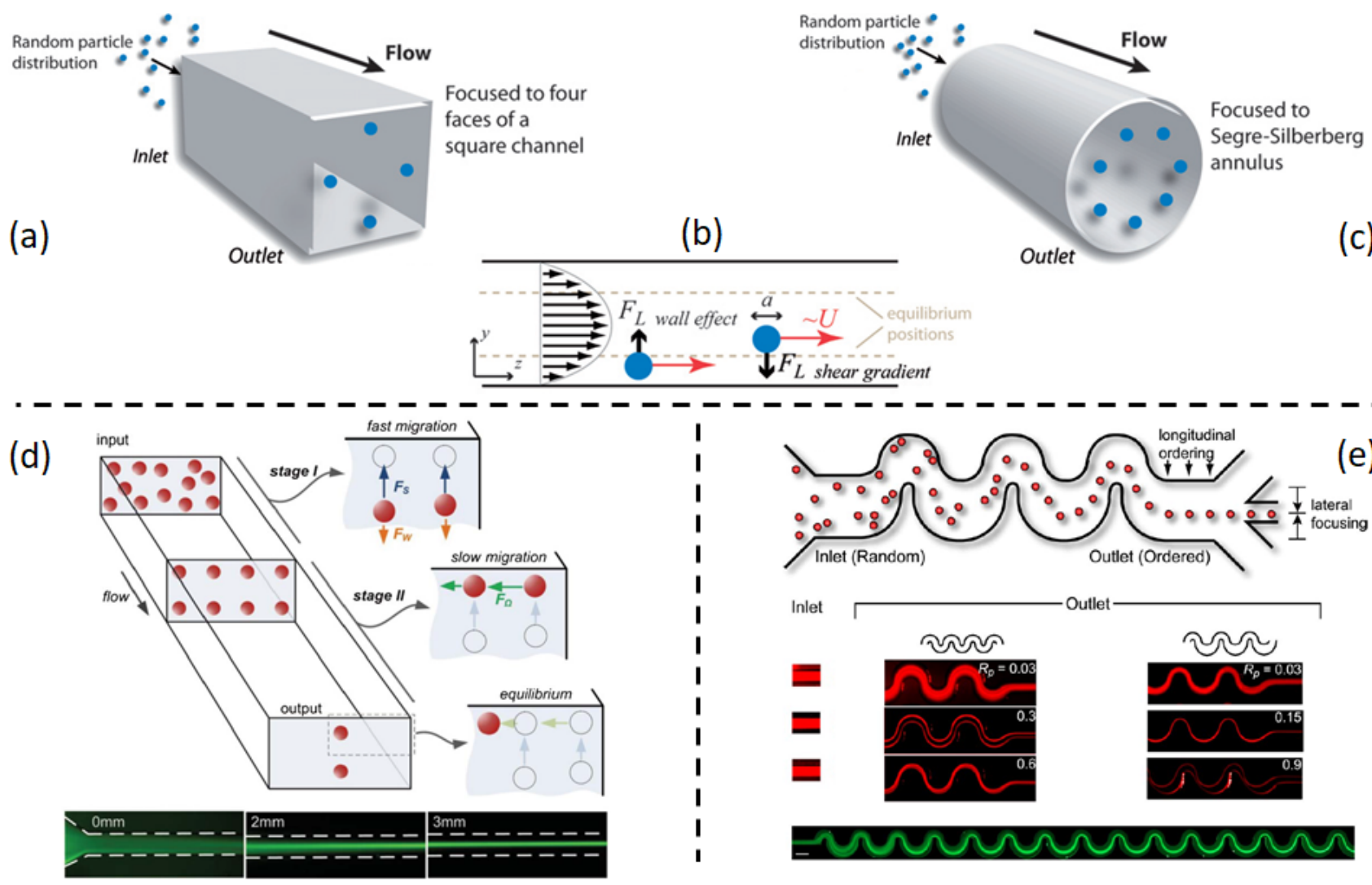

Fig. 7 (a) In a square channel, randomly distributed particles focus to four equilibrium positions located at the center of each edge, while in a cyclindrical channel (c) particles focus to an annulus as a consequence of the two lift forces (b) perpendicular to the flow direction: wall effect lift and shear gradient lift. Adapted from Di Carlo 101 with permission from The Royal Society of Chemistry, copyright 2009. (d) In rectangular channels, two equilibrium positions located at the center of the long edges are observed. The particle cross-stream migration process occurs in two stages: fast migration toward channel walls followed by a slow migration toward the center. Adapted from Zhou and Papautsky 102 with permission from The Royal Society of Chemistry, copyright 2013. (e) Example of inertial focusing in symetrically and asymetrically curved channels and the influence of $R e_{p}$ on its effects. Adapted with permission from Di Carlo et al. .103 , copyright 2007 National Academy of Sciences, U.S.A.

(a)

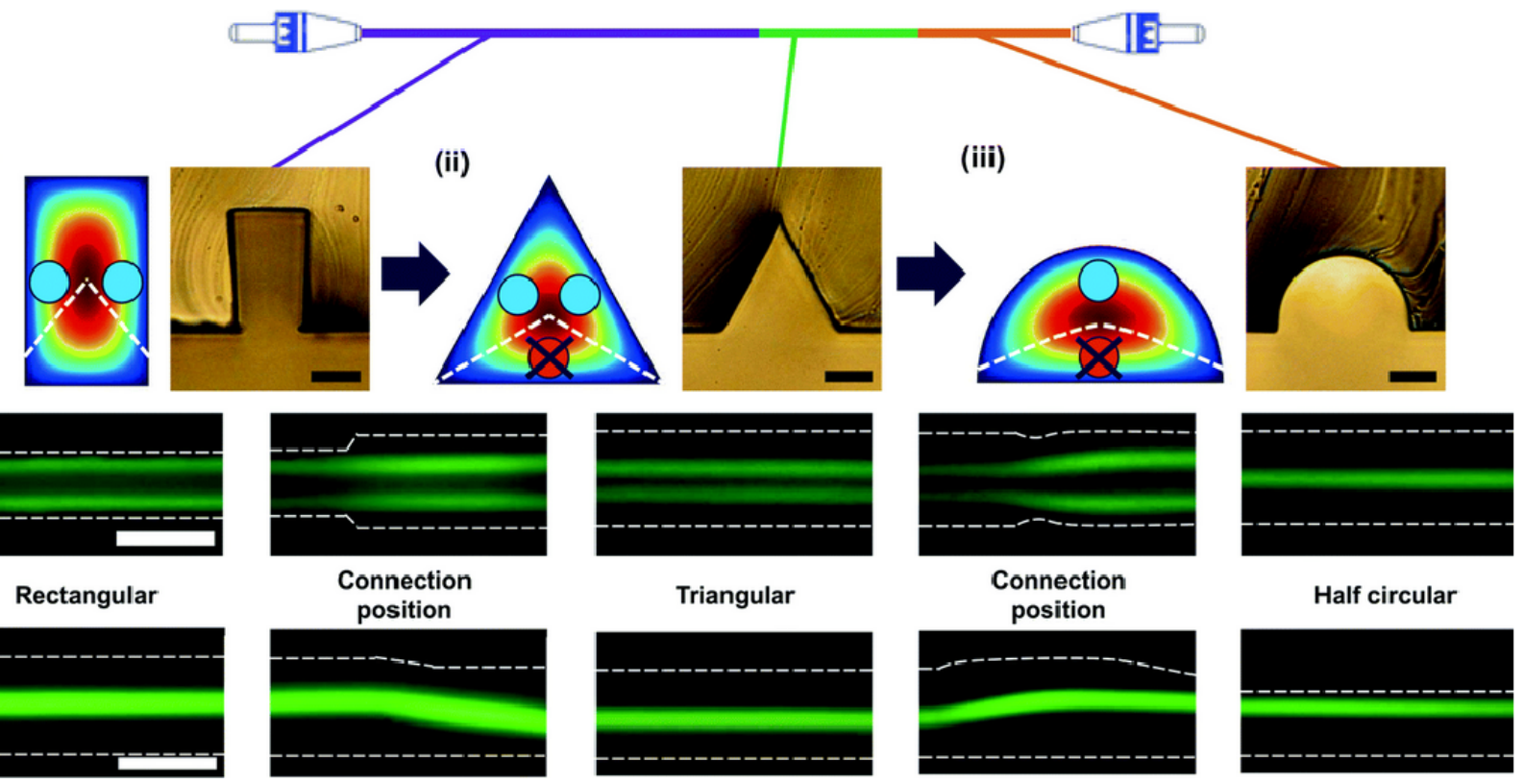

Fig. 8 Inertial single-stream focusing of microparticles in a microchannel of variable cross-sectional shape. (a) The sequence of different cross-sections (rectangular (i), triangular (ii), half circular (iii)) and the respective induced equilibrium positions, makes the particles to be focused in a single stream at the top equilibrium position of the half-circular channel after passing through the whole channel (scale bar $20 \mu \mathrm{m}$ ). Fluorescence images of the top view (b) and side view (c) (scale bar $50 \mu \mathrm{m}$ ). Adapted from Kim et al. 104 with permission from The Royal Society of Chemistry, copyright 2016. 
in two possible equilibrium positions having an equivalent height (Fig. 9a). The comparison of the obtained signal on the same scale (Fig. 9 a vs b) clearly shows a reduction of the signal dispersion.

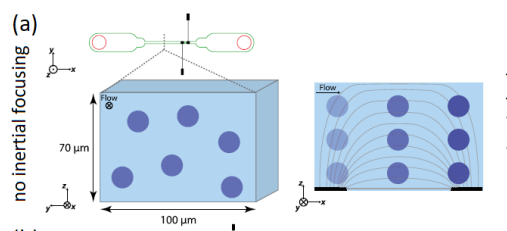

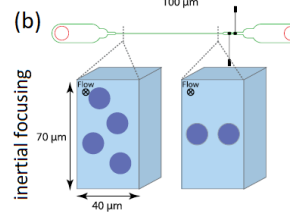

Microfluidic Design

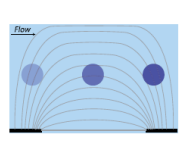

Electrical Field Lines
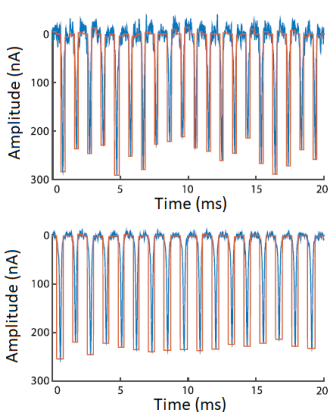

Resulting Electrical Signal
Fig. 9 Illustration of the reduction of the coefficient of variation of the impedance by inertial focusing in the device used by Raillon et al. ${ }^{108}$. (a) Without inertial focusing (b) With inertial focusing. Adapted by permission from Raillon et al. 108 , Wiley, copyright 2019.

The presented device has been tested with several kinds of cells/particles. It demonstrated the ability to count and discriminate 8,15 , and $20 \mu \mathrm{m}$ PS beads suspended in PBS with a reported mean counting error of $1 \%$. As expected because of the dependence of the inertial effects on particle size, the beads were focused on different equilibrium positions, thus experienced different velocities. Three clusters could still be identified from the impedance measurement at $460 \mathrm{kHz}$. The device also successfully differentiated cancer cells from peripheral blood mononuclear cells and RBCs (5\% of false-negative and $0.7 \%$ of falsepositive). Beyond measurements at $460 \mathrm{kHz}$ corresponding to a size information, measuring the impedance at multiple frequencies would provide information on intracellular features $31 / 80 \mid 113$ enabling the identification of CTCs from a background of large White Blood Cells (WBCs) of the same size.

\subsubsection{Dielectrophoretic focusing.}

Dielectrophoresis (DEP) is a technique relying on dipole forces acting on polarizable particles such as biological cells or PS beads subjected to a non-uniform electric field. Following the pioneering works of Pohl and Pethig114|116, DEP has been extensively used for cell/particle separation, trapping and electrical analysis. Several articles review the recent applications of DEP to biological systems $117-120$.

The usually quoted expression for the time-average DEP force acting on a spherical particle of radius $R$ in a medium of absolute permittivity $\varepsilon_{m}$ is $\frac{121}{\text { : }}$

$$
F_{D E P}=2 \pi \varepsilon_{m} R^{3} R e\left(f_{C M}\right) \nabla\left(E_{R M S}^{2}\right),
$$

where $f_{C M}$ is called the Clausius-Mossotti factor, $\nabla$ is the gradient operator, and $E_{R M S}$ is the amplitude (RMS) of the electric field.

The Clausius-Mossotti factor is related to the effective polarization of the particle. It is frequency-dependent and affected by the electrical properties of both the particle and the surrounding medium. The sign of $f_{C M}$ can change according to the medium properties, and to the signal frequency. As a consequence, $F_{D E P}$ can be either in the same direction as the gradient of the electric field, taking particles toward the high electric field regions (positive dielectrophoresis or "pDEP"), or in the opposite direction, moving the particles toward the low electric field regions (negative dielectrophoresis or "nDEP").

It highlights that medium conductivity and signal frequency should be chosen with care to obtain the desired effect. In particular, generating the spectrum of the real part of $f_{C M}$ can be useful 59 . Besides, $F_{D E P}$ is a function of the gradient of the created square electric field (Eq. 97. It is therefore affected by the design and configuration of the electrodes that have a decisive effect on the distribution of the electric field. The voltage applied and electrode material are also important parameters to consider for the efficient running of experimental particle manipulation by DEP 122 .

3.1.3.1 Three-dimensional focusing under nDEP. Particle focusing is done under nDEP. An example of simultaneous lateral and vertical focusing by nDEP is shown in Fig. 10 The design proposed by Morgan et al. ${ }^{123}$ involves two pairs of facing electrodes. The generated electric field exhibits a single point of minimal amplitude located at the center of the channel. Particles are repelled from the edges of the electrodes and moved toward this point under the effect of nDEP. In impedance cytometry, this design has been used for particle focusing upstream of the impedance sensing region 124 . However, extra fabrication steps are required to fabricate parallel electrodes compared to coplanar electrodes requiring a single planar metal layer.

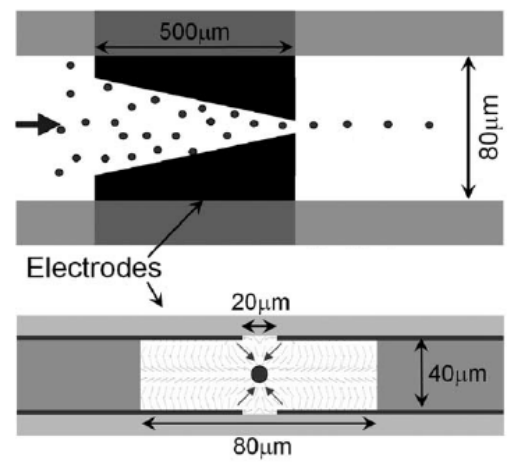

Fig. 10 Example of electrode arrangement for 3D particle dielectrophoretic focusing. Top and cross-section view. Adapted from Morgan et al. .124 with permission from Elsevier, copyright 2006.

3.1.3.2 Lateral nDEP focusing using liquid electrodes. An innovative coplanar electrodes design, also known as "liquid electrodes", used for cell/particle lateral focusing by dielectrophoresis, while keeping a simple fabrication process has been developed by Demierre et al. $\frac{126] 127}{}$. It is based on the fact that microelectrodes fabricated on the bottom of dead-end chambers placed perpendicularly on the side of the main channel provide an homogeneous electric field over the entire channel height (Fig. 11:,d). In other words, this design is similar to having facing electrodes extruded on the walls of the channel. As design rule to obtain the 
(a)
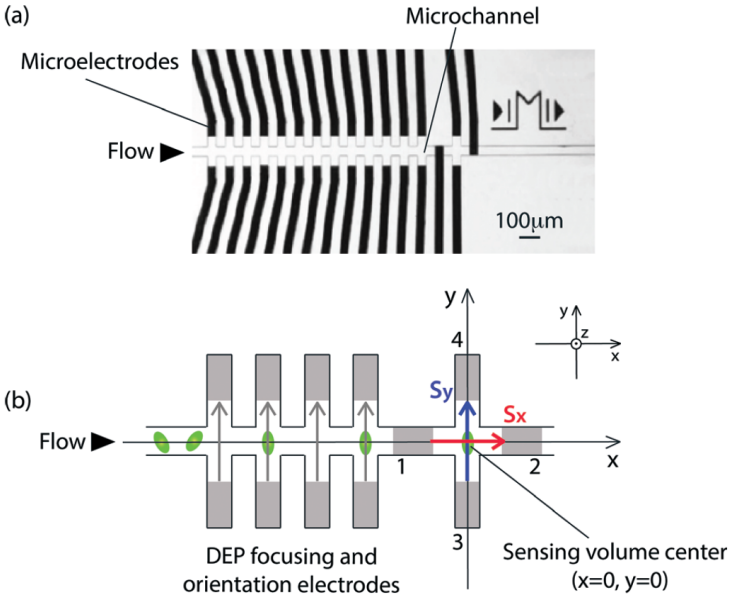

(c)

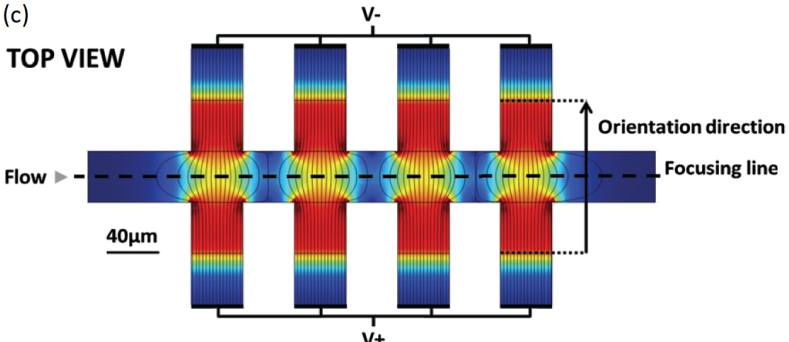

(d)

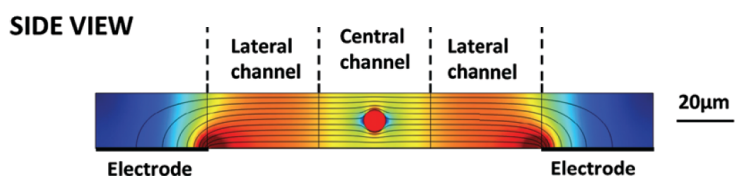

Fig. 11 DEP particle focusing and orientation using several pairs of liquid electrodes upstream of the impedance sensing zone. Adapted from Shaker et al. $\frac{125}{12}$ with permission from The Royal Society of Chemistry, copyright 2014.

quasi independence of the electric field along the vertical direction, it is reported that the distance between the electrodes and the main channel should be equal to the channel height 35 . By this mean, each particle going through the focusing zone is subjected to the same electric field whatever its height. Thus, it has an identical lateral equilibrium position.

Nevertheless, as DEP force scales as the volume of the particle (Eq. 9), it may not be significant enough to deflect rapidly small particles passing at high flow rates. Indeed the movement of the particle resulting from the exposure to the nDEP force takes some time and the particles may reach the end of the focusing region (longitudinal dimension) before reaching the desired streamline (lateral dimension). To overcome this limitation, the number of pairs of electrodes can be increased. For instance, thirteen pairs of facing liquid electrodes were fabricated along the main channel upstream of the impedance sensing region in the device proposed by Shaker et al. for single-cell morphology discrimination 125 (Fig. 11). Moreover, based on numerical simulations performed as part of that work, the authors mentioned an additional vertical focusing effect of the liquid electrodes. This has however not been the focus of a dedicated experimental work so far.

The lateral focusing of $7 \mu \mathrm{m}$ and $10 \mu \mathrm{m}$ PS beads using liquid electrodes has been monitored by Reale et al. 128 . Two impedance sensing units were placed respectively upstream and downstream of the nDEP focusing region. Various conditions of DEP field intensity and flow rate have been successively set, so that different degrees of focusing have been obtained. It turns out that nDEPfocusing cannot work effectively as the particle flow rate rises, whereas increasing particle size is beneficial for its effectiveness.

3.1.3.3 Hybrid DEP focusing. Dielectrophoretic focusing can be combined with other focusing techniques. For instance, the vertical centering of the particle can be done using a pair of facing electrodes, while the lateral focusing is obtained by means of sheath flows 129130 . This is the solution adopted by Evander et al. 130 for an impedance-based platelet analysis. It enables the sample to pass in a single-file along the central axis of the channel through the impedance sensing zone.

\subsubsection{Conclusion on particle focusing methods.}

Focusing methods are used in order to reduce the lateral/vertical position spread of particles in microfluidic electrical impedance cytometers. If the electric field generated in the sensing region is non-homogeneous, particle focusing can limit the variation of the field intensity encountered by the tested particles, thereby reducing the signal dispersion.

The sample stream width and positioning within a large channel can be adjusted by sheath flow focusing. It provides the ability to examine particles with a broad range of size in a single device. However, even if using an insulating sheath fluid also increases the sensitivity of the sensor by reducing the sensing volume, special care has to be taken with the fluid chosen that should not influence the tested particles. More importantly, the main limitations to the use of this method are its complexity and its cost induced by the required additional fluidic systems such as syringe or pressure pumps.

Focusing under nDEP do not require additional buffer inlets and precise flow control. It enables selective control of particles of interest and position adjustment in real-time. It is label-free and the integration of DEP microelectrodes into microfluidic device is relatively easy. On the other hand, this technique does not work effectively as the particle flow rate rises.

Inertial focusing is relatively simple to use and suitable for high-speed applications. It combines a high-throughput with the advantage of requiring no extra instrumentation. However, including no possibility to adapt the focusing position of a given particle, this technique suffers from a lack of versatility. In particular, focusing heterogeneous samples in a single stream remains challenging because of the influence of particle size variations on the lift forces, thus on the equilibrium position. The length needed for a clear particle cross-sectional motion to occur is not negligible and varies with particle size as well. Examples of size heterogeneity within different cell populations are available in the literature $31 / 112132$. In the case of T-lymphocytes, Yang et al.132 reported a radius of $3.29 \pm 0.35 \mu \mathrm{m}$. 

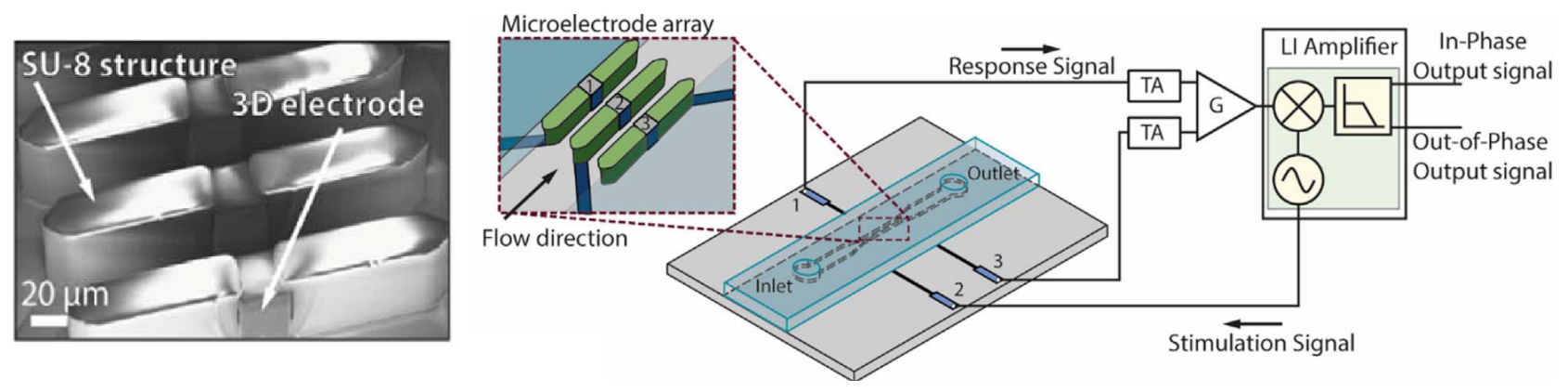

Fig. 12 Two sensing gates composed of three electrodes extruded over channel height. The generated electric field is homogeneous along the vertical axis. Adapted from Rollo et al. 131 with permission from Elsevier, copyright 2017.

\subsection{Focusing-free methods}

Alternatively or in addition to the control of the lateral and/or vertical position of the analyzed cell/particle, the dependence of the measured impedance on the trajectory in the channel can be overcome by focusing-free methods. Some studies focused on designing the sensing zone such that the uniformity of the electric field is enhanced, thereby mitigating the positional dependence.

\subsubsection{Extruded electrodes.}

The extrusion of the microelectrodes composing the impedance sensor over the whole height of the channel is an intuitive way to make the electric field distribution invariant along the vertical axis, and therefore get rid of the dependence of the impedance signal on cell/particle vertical position. The impedance microsensors integrated in the device presented by Rollo et al. 131 consist of vertical platinum electrodes creating multiple probing gates across the channel width (Fig. 12). In each probing gate, the generated electric field exhibits a high uniformity along the channel vertical axis. Moreover, as the gap between the 3D facing microstructures can be adjusted without impacting this uniformity, the impedance dependence on vertical position is decorrelated from the sensitivity. As shown in Fig. 12, the electrodes were extended with passive structures made out of SU-8 that further improve the sensitivity by confining most of the current flow lines in the volume between the facing electrode surfaces. In addition to the measurement of the impedance response of, respectively, $8 \mu \mathrm{m}, 10 \mu \mathrm{m}$, and $15 \mu \mathrm{m}$ diameter beads resulting in coefficients of variation of $4.15 \%, 6.08 \%, 6.89 \%$, the proposed device demonstrated the ability to assess CD8+ lymphocytes activation based on their electrical impedance signature. Measurements variability could be further decreased by ensuring consistent lateral positions of cells passing through the sensing gates. An alternative improvement would be the reduction of the inter-electrode distance that would also benefit the sensitivity at the cost of a reduced range of testable particles and an increased risk of clogging.

\subsubsection{Liquid electrodes.}

Similarly to the extruded electrodes, the liquid electrode design (described above in section 3.1.3.2) reduces the signal dependence on particle height. For this reason, besides its use as particle lateral focusing method, it has also shown to be useful for impedance sensing while keeping a simple fabrication process 35 . In the device presented by Mernier and co-workers 35 , the ob- tained electric field norm variation over the main channel height is less than $3 \%$. A set of simulations was performed placing insulating particles between 5 and $15 \mu \mathrm{m}$ diameter at different vertical position in a $20 \mu \mathrm{m}$ high channel. The amplitude variation of the measured impedance between the highest and the lowest position for a given particle diameter was reported to be between 1\% and $6 \%$. However, the comparison of the proposed microfluidic electrical impedance flow cytometer to classical designs (i.e. facing and coplanar electrodes) showed that the quasi independence on vertical position is obtained at the cost of a reduced sensitivity. The relative impedance variation due to the presence of a 5 to $10 \mu \mathrm{m}$ diameter bead positioned in the center of the channel both vertically and laterally in the proposed device is three to four times lower than in the traditional designs. It can be interpreted as the consequence of the increase of the sensing volume, which includes the volume of the lateral channels.

\subsubsection{Facing electrodes.}

Besides the two outlined solutions for realizing sensors composed of electrodes facing each other, namely 3D microstructures and virtual vertical liquid electrodes, Segerink and co-workers focused on overcoming the complexity of the classical fabrication of parallel electrodes placed at the top and bottom of the channel ${ }^{42}$. The proposed method for the fabrication of top-bottom electrodes involves only one extra processing step compared to the fabrication of planar electrodes. It enables the fabrication and alignment of a floating electrode facing two planar electrodes. Given the floating potential of the upper electrode, there is no need for an electrical connection to both sides of the microfluidic chip. It circumvents the complex packaging step of devices embedding classical facing electrodes.

Even if the field generated by facing electrodes exhibits a better uniformity than the one created in a standard coplanar arrangement, meaning that impedance signal is less dependent on position over the majority of the channel area, it still shows significant variations at extreme positions ${ }^{34}$ (see the simulation files provided as supplementary material ${ }^{\dagger}$ ). In a recently developed device for single-cell dielectric characterization at high-throughput, Spencer and Morgan improved the classical facing electrodes configuration to address this issue (Fig. 13, 56. The electric field distribution has been modified by means of grounded electrodes, resulting in a decrease of the signal variation between center and off-center particles. As illustration, Fig. 13, shows histograms ob- 


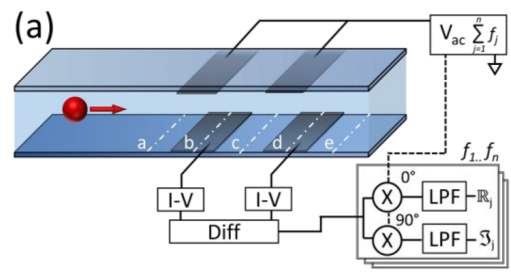

(b)
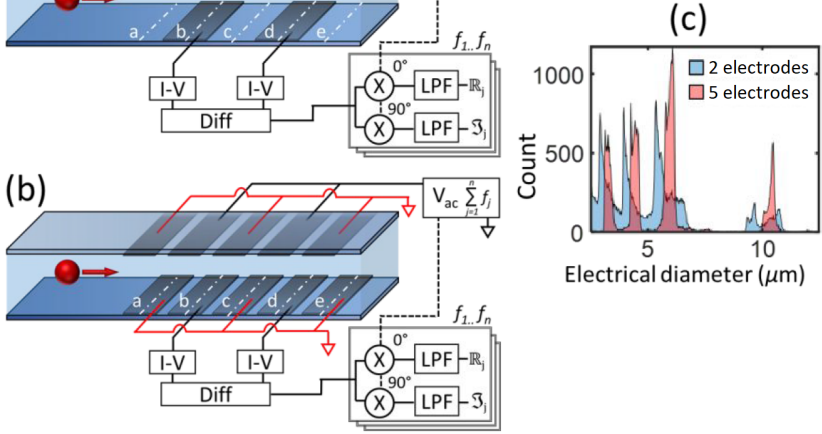

Fig. 13 Illustration of the focusing-free reduction of the coefficient of variation of the impedance by comparison between the conventional facing electrode design and the five pairs of facing electrode arrangement used by Spencer and Morgan [56. Adapted with permission from Spencer and Morgan 56, copyright 2020 American Chemical Society.

tained for a mixture of different PS particles measured using both the conventional facing electrodes and the proposed system. It turns out that particle diameter estimated from the cube root of the low frequency $(500 \mathrm{kHz})$ impedance signal collected using the new design exhibits almost a Gaussian distribution. The corresponding coefficient of variation is similar to the value reported by the manufacturer.

Using multi-frequency impedance measurements, the presented device has been able to determine the conductivity and permittivity of the membrane and cytoplasm of single red blood cells and red cell ghosts (without hemoglobin in the cytoplasm) flowing individually at rates of up to a thousand cells per second.

\subsubsection{Coplanar electrodes.}

Even if it is known to induce a less homogeneous electric field than the facing configuration, some recent works focused on the coplanar configuration that has the advantage of a simple fabrication process.

A finite element analysis of the coplanar electrode layout investigated the influence of the geometrical parameters on the output signal ${ }^{37}$. It highlighted that signal amplitude is almost independent of the height of the tested particle in a $50 \mu \mathrm{m}$ high channel when the inter-electrode gap is $150 \mu \mathrm{m}$, whereas this dependence is very high with a $50 \mu m$ inter-electrode gap. However, the increase of the inter-electrode distance induces a lower peak amplitude as presented in Fig. 14

The work reported by Bilican et al. in 2020 has been done in a similar perspective ${ }^{43}$. In addition to geometrical parameters, the study also regarded the effect of solution conductivity, excitation voltage, and particle size on impedance signal output. It aimed at developing an accurate easy-to-use focusing-free microfluidic impedance-based cell detection system with increased sensitivity. To achieve particle differentiation without particle focusing, the impedance signal had to be made less dependent on particle position. As presented in Fig. 15, fabricating a shallow microchannel (10 $\mu \mathrm{m}$ high) is a way to unambiguously differentiate $3 \mu \mathrm{m}$ from

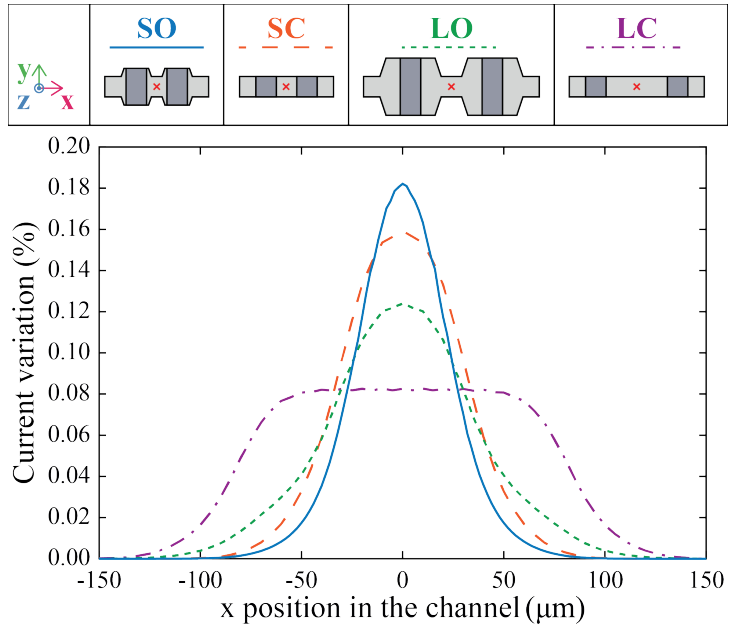

Fig. 14 Simulation of the current variation due to an $8 \mu \mathrm{m}$ diameter polystyrene bead for conventional and optimised coplanar designs with an inter-electrode distance of $50 \mu \mathrm{m}$ (SC and SO) and $150 \mu \mathrm{m}$ (LC and LO). Adapted by permission from Cottet et al. 37 , Springer Nature, copyright 2019.

$6 \mu m$ diameter beads from the measured peak amplitude. Even if the gradient of the electric field is high close to the electrodes, reducing channel height results in a shorter absolute variation of the electric field between the lowest and highest position. For this reason, the generated field distribution is more homogeneous. The efficience of this effect remains a function of the electrode width and gap.

The proposed device was able to experimentally detect and differentiate RBCs and leukemia WBCs using an excitation frequency of $1.5 \mathrm{MHz}$. It proves that precise focusing-free microflu-

a)
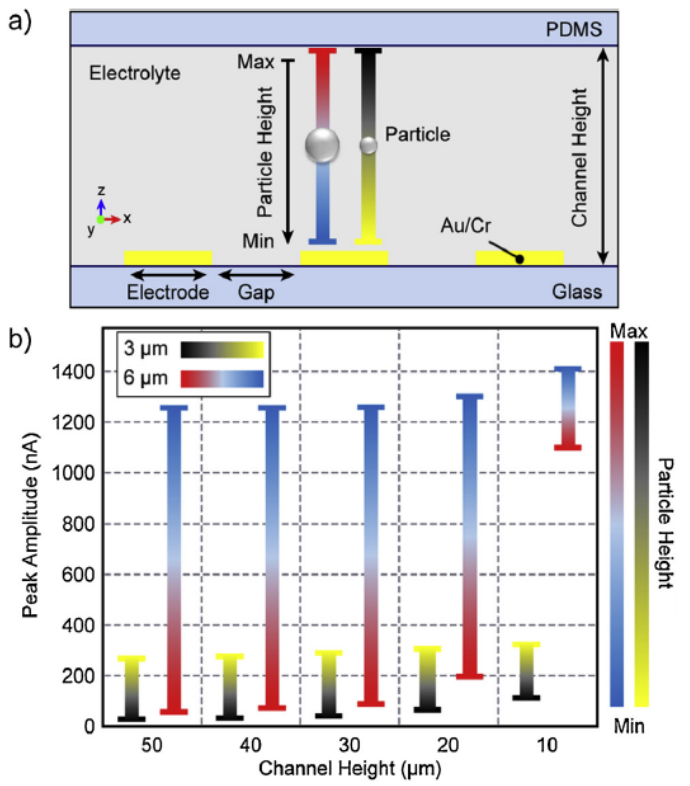

Fig. 15 Illustration of signal variations as a function of channel height. Red-blue gradient bar represents the height range of a $6 \mu \mathrm{m}$ particle; black-yellow gradient bar represents the height range of a $3 \mu \mathrm{m}$ particle. Electrode and gap widths are $10 \mu \mathrm{m}$. Adapted from Bilican et al. ${ }^{43}$ with permission from Elsevier, copyright 2020. 
idic impedance cytometry can be achieved using a simple device integrating coplanar electrodes if the channel dimensions and the size of targeted particles are well-adjusted. However, this strategy increases dramatically the risk of clogging the channel and reduces the range of testable cells/particles. For this reason, it is limited in terms of applications to heterogeneous biological samples.

\subsubsection{Conclusion on focusing-free methods.}

Various techniques have been developed in microfluidic impedance flow cytometry to measure the data accurately without the need for particle focusing. The general principle of the approach consists in mitigating the non-uniformity of the electric field distribution that leads to variations in impedance signals.

It relies on design optimization of the microchannel and/or of the sensing electrodes. This design optimization, supported by the great capability of actual numerical simulation means, allow to generate a more uniform electric field in the sensing zone. By this way, identical particles flowing at different positions through the microchannel create identical impedance signals as they encounter similar field intensity.

Another recently developed focusing-free approach relies on the correction of the impedance data based on either cell/particle position or any metrics reflecting the position. The impedance of single (bio)particles measured at various locations can be related to the impedance that it would have had if it were centered between the sensing electrodes by means of an equation determined from the experimental or modeling results. As impedance signal correction is a typical application of impedance-based integrated position sensors inside microfluidic chips, it will be described in section 4.

\section{Methods to detect cell/particle position}

The impedance dependence on particle/cell position is a wellknown phenomena and numerous solutions have been proposed to tackle this issue, as reviewed in previous sections. Recently, some studies have been published measuring the position in order to compensate or correct the impedance data. In this section, the different techniques that use impedance to measure the position of particles/cells in microfluidic channels will be introduced. They will be compared in terms of methodology, accuracy and throughput when available. Table 1 shows a summary of the studies found that used impedance to measure particles/cells position. The methods were classified in 4 sections: methods using typical coplanar electrodes, facing electrodes, combination of coplanar and liquid electrodes, and new micro-engineered systems.

\subsection{Coplanar electrodes}

As mentioned before, coplanar electrodes are placed side by side on the same wall of the microfluidic channel, producing a non-homogeneous electric field. This phenomenon makes the cell/particle resulting impedance very dependent on the vertical position (Fig. 16, b and c). V. Errico and co-workers ${ }^{39}$ studied this dependence on a 3 coplanar electrodes array where an AC voltage was applied in the middle electrode and the resulting differential current was measured using the other two. In such an
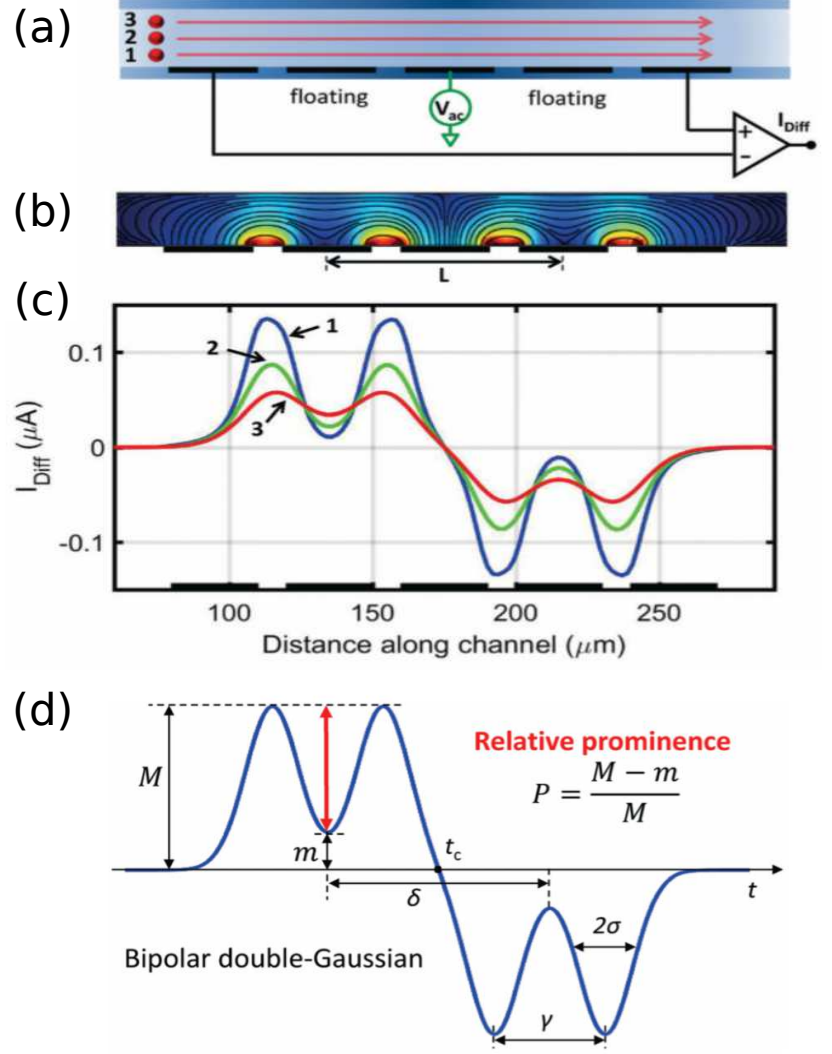

Fig. 16 (a) Particle trajectories at three different altitudes, (b) electric field distribution and (c) differential current obtained and (d) relative prominence parameter used to know the position of the particle for five coplanar electrodes. Adapted from De Ninno et al. ${ }^{133}$ with permission from The Royal Society of Chemistry, copyright 2017.

impedance sensor, the signal resulting from the transit of a particle in the sensing zone exhibits a bipolar Gaussian profile. The experimental data are fitted to this template function by means of four parameters. Correlations can be found between these parameters and particle velocity, particle height, as well as particle diameter. In particular, particle velocity is deduced from particle transit time (i.e. peak-to-peak time). Similarly, particle height is correlated with peak width normalized by the transit time, and particle nominal diameter is given by a simple function of peak amplitude, and peak width together with the transit time (reflecting particle height). Using this technique, the authors could mitigate the influence of the positional dependence of the data, by correcting the signal according to particle/cell trajectory. In particle sizing applications, they obtained coefficients of variation (CV) similar to studies using particle focusing systems. Moreover, this system also provides the ability to discriminate beads from yeasts despite the similar size, as well as two yeast populations. It has been reused in 2019 by De Ninno et al. 134 for highthroughput label-free discrimination of viable, necrotic and apoptotic human lymphoma U937 cells. This work exploited multifrequency measurements together with impedance data correction using an impedance-based metric correlated with cell height.

The presented design was extended to 5 coplanar electrodes $133 \mid 135$. As presented in Fig. 16, an AC voltage was ap- 
plied in the middle (3rd) electrode, the adjacent electrodes were set as floating electrodes, and the differential current was measured between the first and last electrodes. The output signal of the sensor caused by the passage of a flowing particle exhibits a bipolar double-Gaussian profile that can be fitted using five parameters. Integrating a floating electrode at each side of the excitation electrode lead to the duplication of the signal shape obtained by the 3 electrodes design. As shown in Fig. 16 d, the so-called relative prominence can be extracted from the fitted signal. This metric turned out to be strongly correlated with particle trajectory height $\frac{135}{}$. The relative prominence is thus a suitable metric to estimate particle vertical position. Moreover, being an impedance-based indicator of particle position, it can be used to correct the impedance signal without the need for optical position feedback. Both devices were able to correct the signal of mixed 5,6 and $7 \mu \mathrm{m}$ diameter PS particles. Almost gaussian size distributions have been obtained with CVs similar to those given by the suppliers. The comparison of the 3 and 5 electrodes layouts shows slightly smaller CVs for the latter. However, this reduction is obtained at the cost of an increased risk of coincident detection because of the longer sensing zone. It is worth mentioning that experimental parameters such as electrode material, dimensions of the system, signal frequency and electrolyte conductivity influence the relationship between prominence, particle height and particle diameter 135 . Therefore, a calibration with known particles should be done to every device. This calibration remains valid as long as the measurements are done in steady experimental conditions.

In parallel with these works, a device integrating a pair of coplanar star-shaped electrodes has been developed for the purpose of precise real-time position sensing along the microfluidic channel. Unlike the above-mentioned strategy relying on fitting the experimental data to a template function, thus providing a position estimation after the transit of the tested particle through the sensing zone, Brazey et al. $\frac{136}{16}$ focused on estimating the position of a particle in real-time as long as it is present in the sensing zone. The star shape was chosen in order to avoid a plateau in the current measured when the particle is centered between the electrodes. By this way, any particle motion gave rise to a current variation which was combined with an approximate knowledge of particle velocity by a Kalman filter enabling position estimation. This allowed the authors to monitor the $80 \mu \mathrm{m}$ path of a $8.7 \mu \mathrm{m}$ particle between the two electrodes composing the sensor. The maximun error on position estimation was less than $5 \mu \mathrm{m}$. No application of this system to impedance data correction within the scope of particle/cell characterization has been reported so far. Since a model of the impedance variation as a function of position is needed by the Kalman filter to estimate the real-time position from a provided impedance measurement, the application of this approach to heterogeneous biological samples would require the ability to adapt the model according to the particle in the sensing zone.

\subsection{Facing electrodes}

As seen in section 2.3 the electric field distribution between facing electrodes arrays is more homogeneous than for the coplanar, nevertheless, position particle still affects the impedance measured. This is due to both the non-homogeneity of the electric field and the diagonal current flow when using two or more pairs of electrodes in differential measurement configuration. To overcome this issue, a study introduced a 10 electrodes array among which 4 electrodes were grounded (Fig. 17a). An AC voltage was applied to 2 electrodes, and the differential current was measured from the other 4 electrodes 137 . This special configuration enables the measurement of two differential currents: transverse
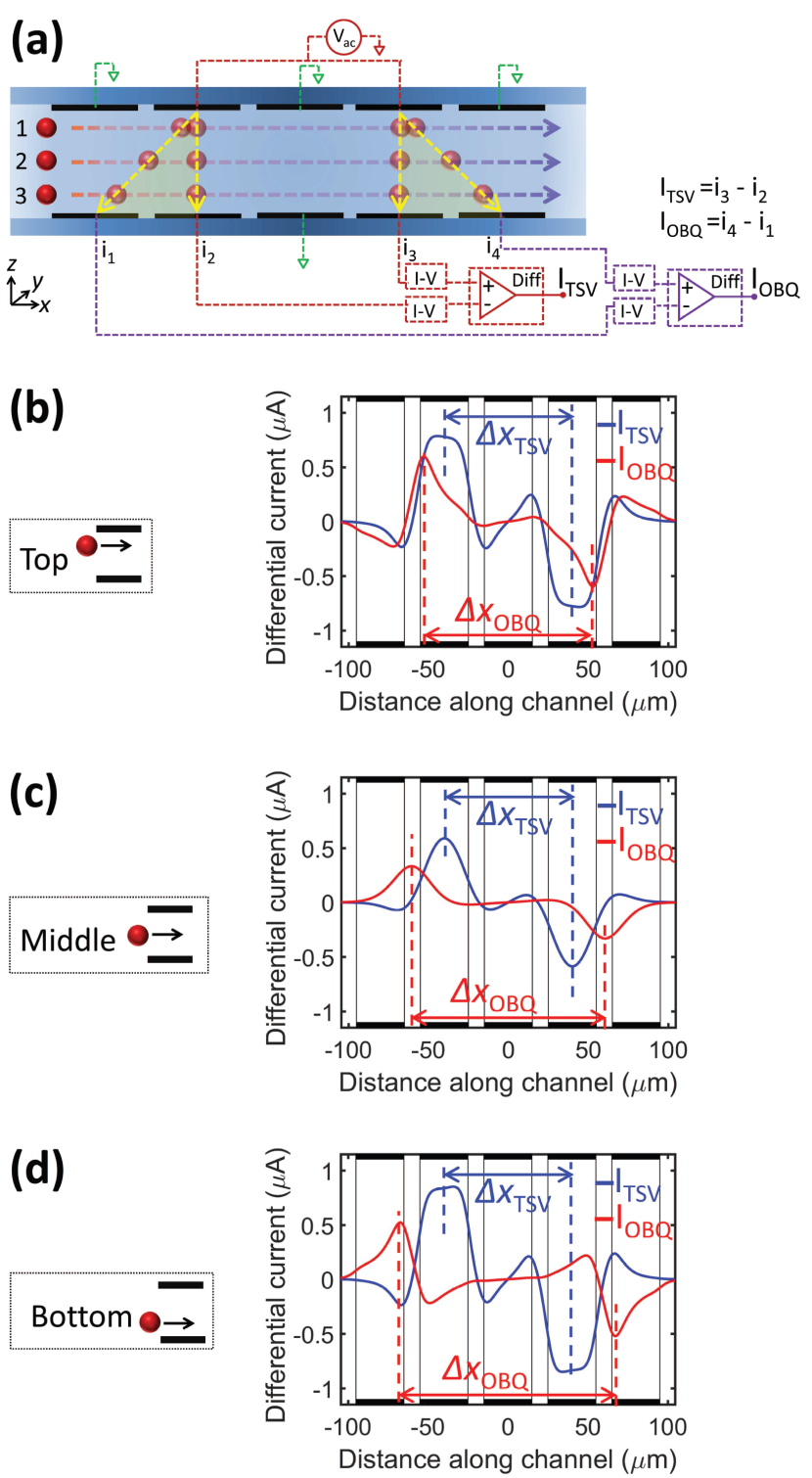

Fig. 17 (a) 10 facing electrodes enabling transverse and oblique differential currents measurement. Resulting signals from the transit of a particle at the top (b), middle (c), and bottom (d) of the channel. Peak-to-peak time in transverse current (blue curve) can be used to deduce particle velocity, as it does not depend on particle height, whereas the peak-to-peak time in oblique current (red curve) does. The ratio of both differential currents can be related to particle height. Adapted from Spencer et al. 137 with permission from The Royal Society of Chemistry, copyright 2016. 
and oblique. The peak-to-peak time in the oblique current depends on particle height (Fig. 17p, c and d). It can be normalized by the time that a particle takes to travel from center to center of the excitation electrodes (i.e. the peak-to-peak time in the tranverse current) which is independent of particle trajectory and reflects particle velocity. The obtained normalized metric was used to deduce the height of the cell/particle. By fitting the data with a quadratic equation dependent on 3 experimentally obtained parameters, the signal could be corrected obtaining very similar particle size (5, 6 and $7 \mu \mathrm{m}$ ) CVs as the manufacturers quoted values.

In order to reduce the number of electrodes, thus simplify the system, F. Caselli and co-workers ${ }^{40 \mid 139}$ introduced a new wiring scheme composed of two pairs of facing or liquid electrodes. This

(a)

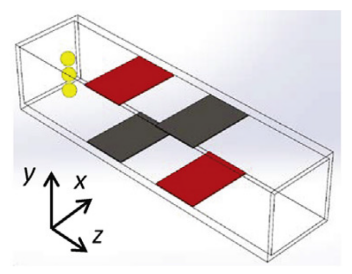

(b)

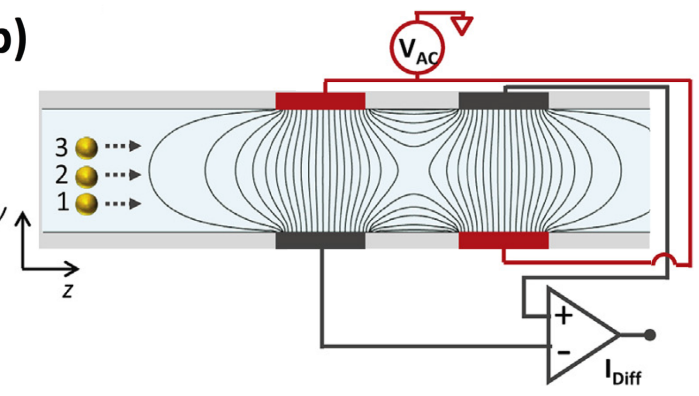

(c)

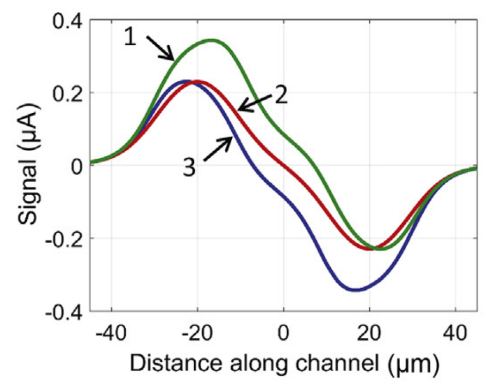

(d)

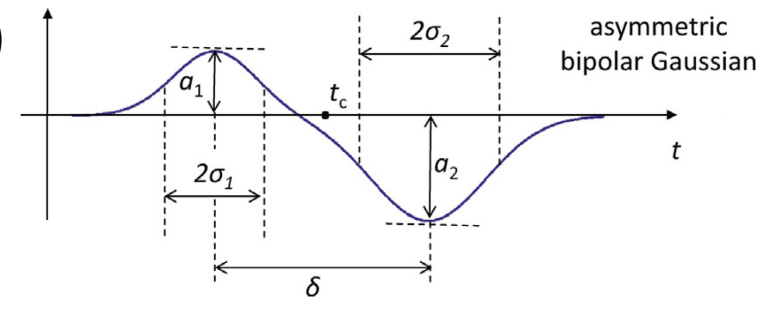

Fig. 18 (a) New wiring scheme for facing electrodes proposed by Caselli et al. 40 and (b) resulting electric field lines. (c) Signals obtained from the transit of of a particle at the top (blue line), middle (red line), and bottom (green line) of the channel. (d) Asymmetric bipolar gaussian function to which the experimental data are fitted. Adapted from Caselli et al. 40 with permission from Elsevier, copyright 2018. wiring configuration consists in applying an AC voltage to diagonally opposite electrodes and measuring the current on the remaining ones (Fig. 18,b). Similarly to previous studies, the signal was fitted to a bipolar Gaussian equation. However, this one is asymmetric, meaning that the amplitude and width of the positive and negative peaks are not equal, unless the tested (bio)particle passes at the middle of channel height (Fig. 18,d). A new metric correlated to particle height is introduced, namely the pulse amplitude relative difference. It enables the compensation of impedance positional dependence in cell/particle sizing applications, obtaining very similar CVs to the size distribution of the particles (5.2, 6 and $7 \mu \mathrm{m}$ in diameter). In 2019, this method was also used to measure the position of particles before and after DEP focusing 128 . More recently, Honrado et al. 140 used the new wiring scheme in two consecutive facing pairs of electrodes to measure both the lateral position and the height. The implementation of artificial intelligence approaches instead of classical curve fitting helped to fasten dramatically the extraction of the size, cross-sectional position and velocity from the data obtained.

\subsection{Combination of planar and liquid electrodes}

Recently, some studies introduced a combination of the previously presented methods in order to obtain the 3D position of cells/particles, as presented in Fig. 19 . In particular, Reale et al. 45 used a set of 5 planar electrodes crossing the microfluidic channel (Fig. 19k) to estimate the height of the flowing particles. Indeed, the ouput signal resulting from the passage of a particle has the same shape as the one obtained using the design proposed by De Ninno et al.133 (see section 4.1 above). Therefore, the vertical position of the flowing particles can be deduced from the correlated relative prominence exhibited by the experimentally obtained signal. This has been combined with an upstream set of 10 planar liquid electrodes (Fig. 19p) enabling the measurement of the lateral position. Since liquid electrodes work as virtual extruded electrodes facing each other (see description of liquid electrodes principles in section 3.1.3.2 above), the technique presented by Spencer et al. $\frac{137}{}$ (explained in section 4.2 above) using five pairs of facing electrodes has been easily adapted and reused in that new device.

In a more recent study, Reale et al.141 used the same approach to measure cell/particle height but combined it with the method and wiring scheme developed by Caselli et al. 40 (see section 4.2 above) for the measurement of the lateral position. The lateral position measurement area of the proposed device thus integrated just 2 pairs of liquid electrodes instead of 10 . The described system has been used to detect the position of nonspherical cells. This approach proved to have the potential to be used in cytometry studies of red blood cells.

\subsection{New microengineered systems}

Another approach to measure the position of cells/particles in microfluidic channels using impedance is the engineering of new

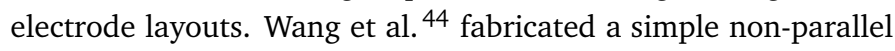
coplanar pair of electrodes (Fig. 20a). When a particle flows next to the section where the electrodes are closer to each other (top 


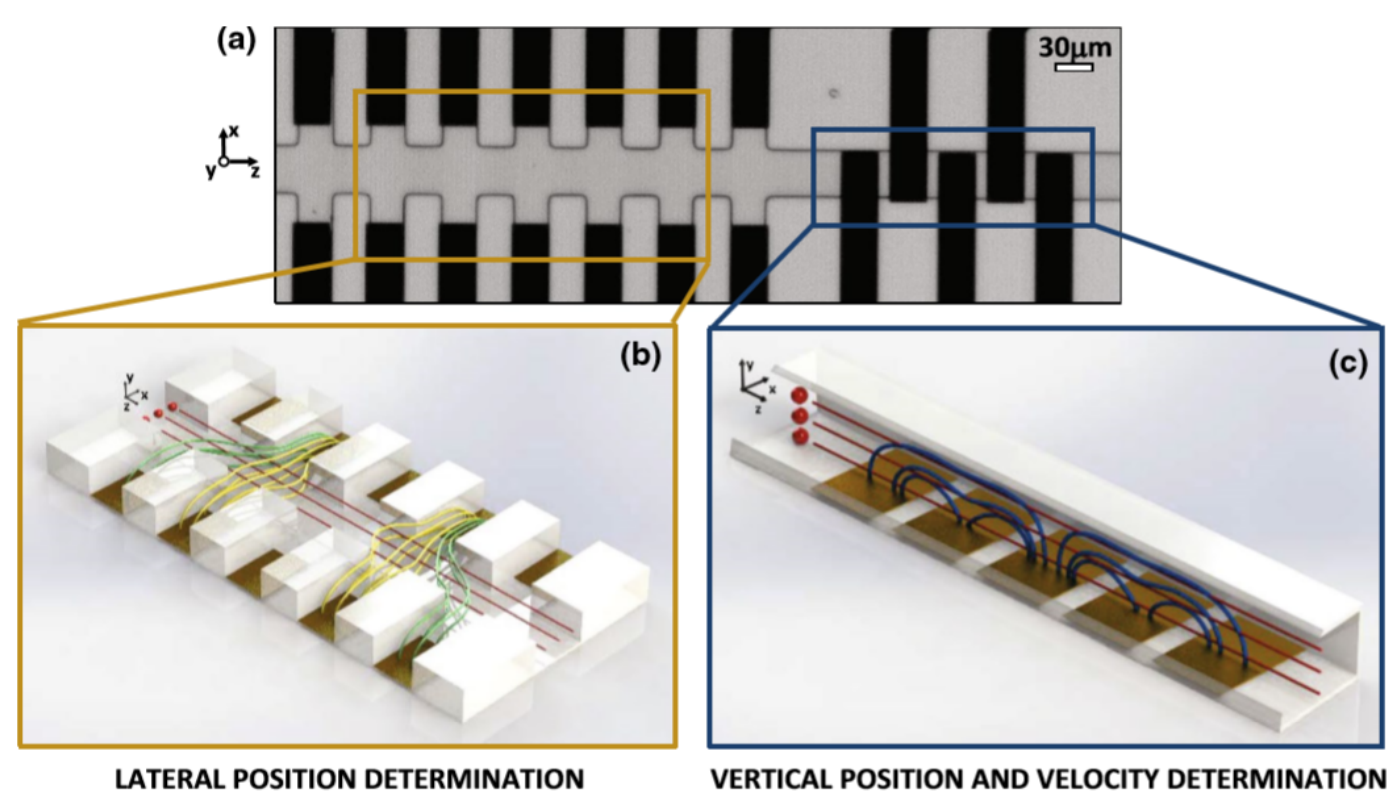

Fig. 19 (a) Top-view of the electrode array. (b) Drawing of the five pairs of liquid electrodes used to determine the lateral position and (c) drawing of the five coplanar electrodes used to determine the height and velocity of the particles. Reproduced from Caselli et al. .45

(a)
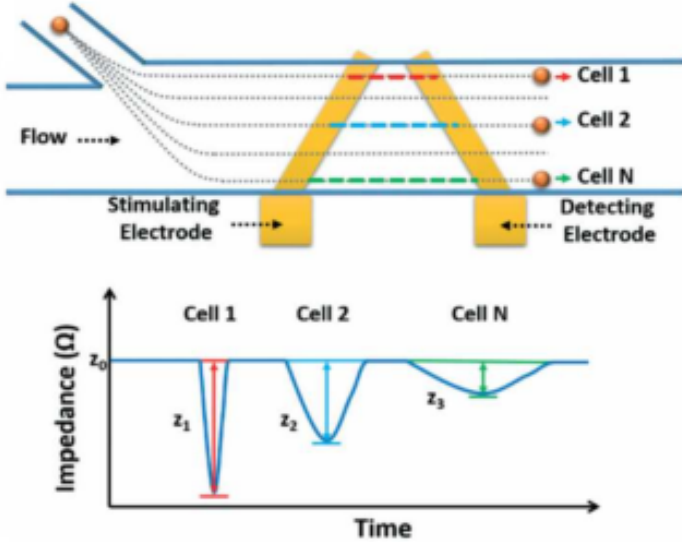

(c)

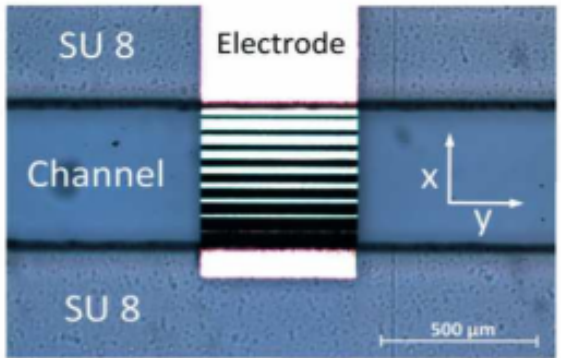

(b)

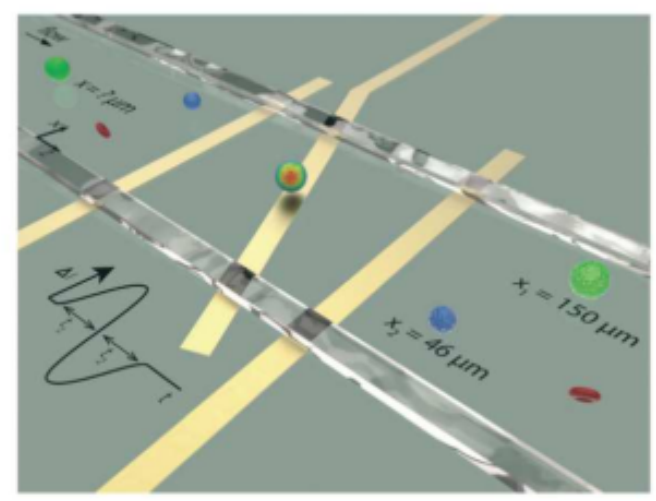

(d)

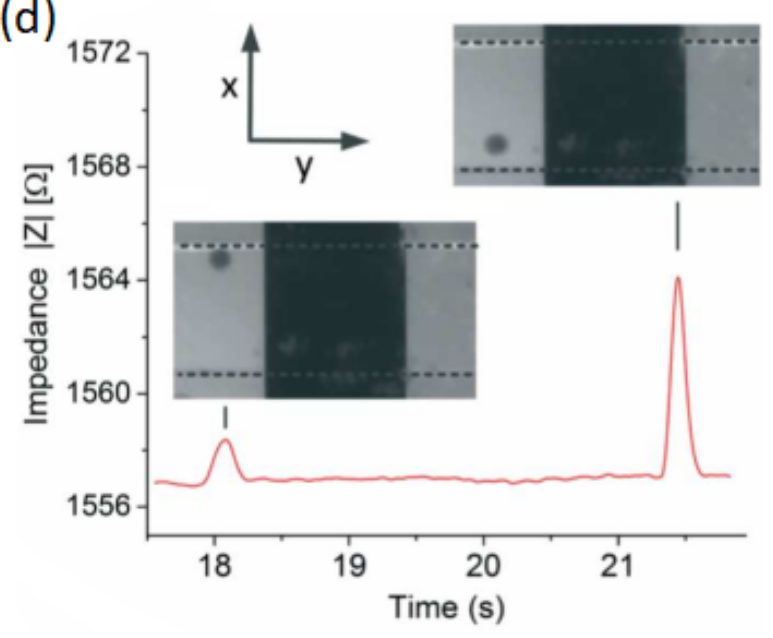

Fig. 20 (a) V-shaped coplanar electrodes used to measure the lateral position and simulated signal obtained in 3 different trajectories. Reproduced from Wang et al. ${ }^{44}$ with permission from The Royal Society of Chemistry, copyright 2017. (b) N-shaped coplanar electrodes that perform a nonsymmetrical differential current measurement. Reproduced from Yang and Ai ${ }^{138}$ with permission from The Royal Society of Chemistry, copyright 2019. (c) Increasing electrodeposited area from top to bottom on gold facing electrodes, (d) and signal produced when particles flow at the top and bottom of the channel. Adapted from Solsona et al. $\frac{46}{6}$ with permission from The Royal Society of Chemistry, copyright 2019. 
part in Fig. 20a), there is a higher electric field, and a larger and thinner current peak is measured compared to the peak obtained after the transit of a particle in the lower part of the microchannel. By varying the angle of separation between the electrodes the authors could detect the lateral position of PS particles 6 and $11 \mu \mathrm{m}$ in diameter with a position resolution of $33 \mu \mathrm{m}$ (20\% of the total channel width) at a speed of $0.01 \mathrm{~m} . \mathrm{s}^{-1}$.

Yang and $\mathrm{Ai}^{138}$ developed a three non-parallel electrodes array (Fig. 20p) to measure the lateral position of cells/particles flowing in a microfluidic channel. An AC voltage of $3 \mathrm{~V}$ and $500 \mathrm{kHz}$ was applied and the differential current was measured in the middle and outside electrodes respectively. This method is similar to the previous one, however, it performs a differential current measurement, which enhances the sensitivity of the system. This innovative electrode configuration allowed the authors to reach a $5.15 \%$ position resolution, to use smaller particles $(3.6 \mu \mathrm{m})$ and to work at very high speeds (up to $0.21 \mathrm{~m} . \mathrm{s}^{-1}$ ).

Solsona et al.46[142, used a gradient in electric field to measure the lateral position of particles flowing in a microfluidic channel. The gradient was formed when applying an AC voltage on an increasing porous area in a 2 facing electrodes array (top to bottom in Fig. 20, ), which were electrodeposited with black platinum on gold electrodes. The porous areas increased the double layer capacitance (CDL) therefore decreasing the impedance in those regions at relatively low frequencies (Fig. 20d). With this method they were able to measure the position of big particles (80 $\mu \mathrm{m}$ in diameter) with a resolution of $12.5 \%$ and measure the conductivity of the system at high frequencies.

\subsection{Conclusion on methods to detect cell/particle position}

During the last five years the innovation of different research groups has demonstrated the possibility to measure and compensate variations of particle position in impedance cytometry studies. Some have shown that standard planar or facing electrode configuration enables the measurement of cell/particle position and the final data correction. Other microfabricated new electrode designs have been proposed in order to enhance the positional dependence and therefore make its detection easier.

Most of the presented devices have shown the ability to deal with size heterogeneous samples. Beyond that, it is also possible to provide cell/particle position and accurate characterization at the same time $45,138[140] 141$.

\section{Discussion and Outlook}

Over the last two decades, microfluidic electrical impedance flow cytometry has shown its ability to discriminate cells on the basis of morphological characteristics (size and shape), but also of subcellular features, such as membrane structure, cytoplasm conductivity and nucleus size. This study focuses on the variation of the impedance signal as a function of the trajectory of (bio)particles in the microchannel. In the context of accurate cell characterization for identification, counting and/or sorting purposes, the interest of carefully considering this variation has been highlighted. In addition to its impact in single-cell analysis, the positional dependence of the impedance signal has been shown to be an op- portunity for the development of integrated impedance-based position sensors.

\subsection{Single-cell counting and analysis}

As shown in Fig. 2 and 3 , the variation of the impedance signal as a function of cell trajectory is highly dependent on the design of the sensing zone, and can be the cause of sorting errors and of unexpected spreads in cell populations extracted features. So far, this impact on measurements has mostly been highlighted in the standard coplanar and facing electrode configurations $34|39| 56 / 133 \mid 137$. However, the positional dependence is not restricted to those configurations. Future non-conventional designs may induce a greater positional dependence.

Researchers are encouraged to simulate the transit of particles along different trajectories within the designed sensing region (see section 2.2, section 2.3 and the supplementary material ${ }^{\dagger}$ for a detailed method). If the variations of the obtained output signals are significant compared to the natural heterogeneity of the analyzed biological samples, the use of a method to reduce the impact of the positional dependence of impedance measurements is beneficial for the accuracy of the system. None of the reviewed particle focusing strategies provides ease of use, design simplicity, ease of fabrication, low cost instrumentation and versatility at the same time. For this reason, future microfluidic impedance cytometers are expected to rather make use of focusing-free methods to perform accurate particle characterization. The impact of the positional dependence can either be corrected by signal processing based on a position feedback, or mitigated without extensive signal correction by designing the sensing zone such that the uniformity of the electric field is improved.

In some cases, according to the degree of heterogeneity of the population of interest, the reduction of the dispersion of the impedance data among each subpopulation may still not be enough to distinctly separate them. In other words, the mitigation of the impact of particle position on the measured impedance is not sufficient to completely remove the overlap of the data from different subpopulations 134 . Multi-frequency measurements can be done to provide informations on the dielectric properties of several components of the cells in addition to its morphological features 113 . This way, the probability to obtain a parameter differing significantly from one subpopulation to another is increased, thereby reducing the risk of identification errors.

In addition to considering the positional dependence, toward the final goal of providing an easy-to-use, accurate, low-cost, label-free, high-throughput cell analysis system, the next generation of microfluidic impedance cytometers is envisioned to consider coincidence detection (i.e. several particles passing through the sensing zone simultaneously) 143 . This limitation for the throughput and accuracy of the devices can be physically avoided by longitudinal focusing of particles using different channel topologies ${ }^{144}$ or numerically resolved by means of a Bayesian approach enabling the decomposition of signals generated by coinciding particles into individual particle contribution ${ }^{145}$.

Moreover, the use of artifical intelligence techniques recently proved to be useful to increase the throughput of impedance 


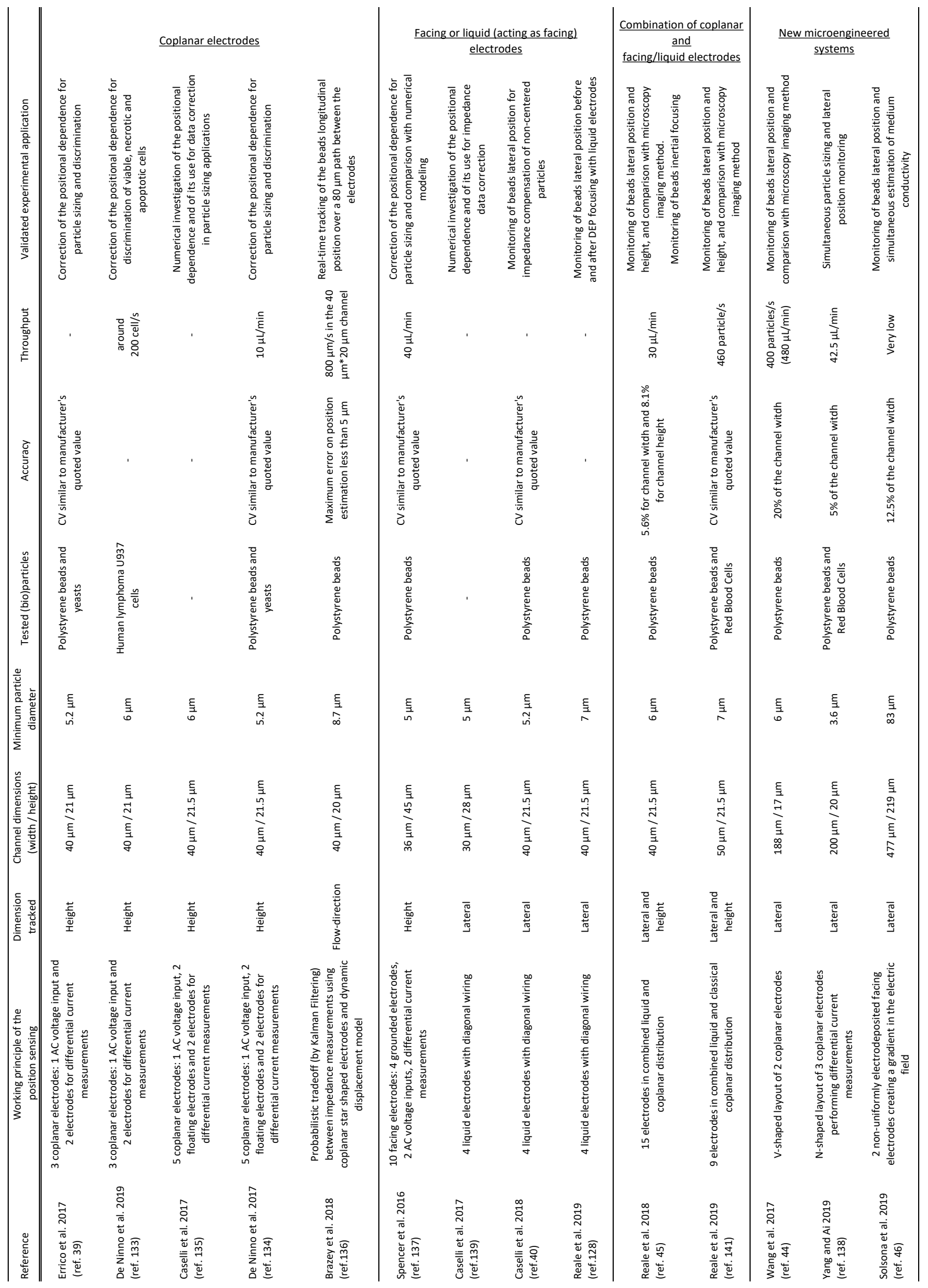


flow cytometers. Neural networks have been implemented for cell characterization instead of classical template curve fitting. It fastened dramatically the feature extraction of the tested cells, allowing real-time characterization 140 .

Overall, the future development of microfluidic impedance cytometers is likely to open the door to faster and more selective label-free single-cell analysis. Beyond biomedical applications, the developed technologies are attractive in a large range of domains that also involve counting and identification of small particles such as material science, food industry, and water quality.

\subsection{Impedance-based position sensing}

So far, the most common way to detect particle position at the microscale is based on optical means. While conventional video microscopy projects particle trajectory onto the focal plane of the objective, numerous optical methods have been developed enabling particle tracking in three dimensions (for a full description see the review ${ }^{146}$ ). However, those methods turn out to require expensive and complex optical instrumentation, and/or demanding image processing 147 . In addition to the complex image processing, the sampling frequency of the camera (up to a few kilohertz in the case of expensive fast cameras) limits the ability of visionbased methods to provide fast position information. Moreover, those methods require specific lighting conditions to provide sufficiently contrasted images in spite of the short exposure time. Besides, microscopes have a limited field of view that restricts the area over which a position information is available at a given time point.

The development of integrated impedance-based position sensors has been made possible by exploiting the positional dependence of the impedance signal. Recent applications prove that, beyond its classical use in microfluidic devices for cell characterization purposes, electrical impedance is a credible alternative to vision as a way to detect particle position. In particular, impedance-based position detectors provide an accurate position estimation and are easy to use in experimental conditions. Just the expression linking the electrical measurements to the position is needed. It can be obtained by experimental calibration. Moreover, having a sampling frequency up to hundreds of megahertz followed by a straightforward signal processing, impedancebased position sensors are suited to applications requiring position information at high speed.

In the context of electrical impedance single cell analysis, it has been shown that correcting signal variations due to the positional dependence can improve the accuracy of the system. To provide the position information required by this correction, impedance-based position sensors may be integrated within microfluidic impedance cytometers instead of external optical setup. Providing a real-time correction of the data according to particle position would make an increase of the possible flowrates. To further increase the throughput of the device, single-cell electrical assays with integrated position correction could even be performed in parallel in tens of microchannels. Such a parallelization would be beyond the field of view of a classical microscope, thus making signal correction impossible.
Overall, getting rid of the optical setup opens the door to the development of commercial simple and low-cost plug-and-play microfluidic chips for high throughput accurate single-cell characterization with integrated signal correction. Besides microfluidics, having accurate and fast position feedback is essential in the field of microrobotics. The development of impedance-based position sensors is the opportunity to obtain real-time position feedback for micromanipulation tasks 148,150 .

\section{Conflicts of interest}

The authors declare no conflicts of interest.

\section{Acknowledgements}

This work has been supported by the EUR EIPHI program (Contract No. ANR-17-EURE-0002), by the French Agence Nationale de la Recherche and the Swiss National Science Foundation through the CoDiCell project (contract "ANR-17-CE33-0009" and "No. 00021E_175592/1", respectively), by the MiMedi project funded by BPI France (grant No. DOS0060162/00) and the European Union through the European Regional Development Fund of the Region Bourgogne-Franche- Comte (grant No. FC0013440), by the Collegium SMYLE (SMart SYstems for a better LifE).

\section{References}

1 C. Alix-Panabières and K. Pantel, Cancer Discovery, 2016, 6, 479-491.

2 M. Wildgruber, T. Aschenbrenner, H. Wendorff, M. Czubba, A. Glinzer, B. Haller, M. Schiemann, A. Zimmermann, H. Berger, H.-H. Eckstein, R. Meier, W. A. Wohlgemuth, P. Libby and A. Zernecke, Scientific Reports, 2016, 6, 1-8.

3 D. R. Gossett, W. M. Weaver, A. J. Mach, S. C. Hur, H. T. K. Tse, W. Lee, H. Amini and D. Di Carlo, Analytical and Bioanalytical Chemistry, 2010, 397, 3249-3267.

4 O. G. Martinsen, S. Grimnes and H. P. Schwan, Encyclopedia of surface and colloid science, 2002, 20, 2643-2653.

5 Y. Feldman, I. Ermolina and Y. Hayashi, IEEE transactions on dielectrics and electrical insulation, 2003, 10, 728-753.

6 V. Raicu and Y. Feldman, Dielectric Relaxation in Biological Systems: Physical Principles, Methods, and Applications, Oxford University Press, 2015.

7 H. P. Schwan, Annals of the New York Academy of Sciences, 1999, 873, 1 .

8 K. Asami, Progress in Polymer Science, 2002, 27, 1617-1659.

9 T. Hanai, Kolloid-Zeitschrift, 1960, 171, 23-31.

10 T. Hanai, K. Asami and N. Koizumi, Bulletin of the Institute for Chemical Research, KyotoUniversity, 1979, 57, 297-305.

11 C. Boned and J. Peyrelasse, Colloid and Polymer Science, 1983, 261, 600-612.

12 S. J. Altschuler and L. F. Wu, Cell, 2010, 141, 559-563.

13 W. M. Weaver, P. Tseng, A. Kunze, M. Masaeli, A. J. Chung, J. S. Dudani, H. Kittur, R. P. Kulkarni and D. Di Carlo, Current Opinion in Biotechnology, 2014, 25, 114-123.

14 J. R. Heath, A. Ribas and P. S. Mischel, Nature Reviews Drug Discovery, 2016, 15, 204-216. 
15 D. Gao, F. Jin, M. Zhou and Y. Jiang, Analyst, 2019, 144, 766-781.

16 G.-C. Yuan, L. Cai, M. Elowitz, T. Enver, G. Fan, G. Guo, R. Irizarry, P. Kharchenko, J. Kim, S. Orkin, J. Quackenbush, A. Saadatpour, T. Schroeder, R. Shivdasani and I. Tirosh, Genome Biology, 2017, 18, 84.

17 G. M. Whitesides, Nature, 2006, 442, 368-373.

18 E. K. Sackmann, A. L. Fulton and D. J. Beebe, Nature, 2014, 507, 181-189.

19 P. Cui and S. Wang, Journal of Pharmaceutical Analysis, 2019, 9, 238-247.

20 R. Burdett, Handbook of Measuring System Design, Wiley, 2005.

21 H. Morgan, T. Sun, D. Holmes, S. Gawad and N. G. Green, Journal of Physics D: Applied Physics, 2007, 40, 61-70.

22 T. Sun and H. Morgan, Microfluidics and Nanofluidics, 2010, 8, 423-443.

23 K. C. Cheung, M. Di Berardino, G. Schade-Kampmann, M. Hebeisen, A. Pierzchalski, J. Bocsi, A. Mittag and A. Tárnok, Cytometry Part A, 2010, 77A, 648-666.

24 J. Chen, C. Xue, Y. Zhao, D. Chen, M.-H. Wu and J. Wang, International Journal of Molecular Sciences, 2015, 16, 9804 9830.

25 Y. Xu, X. Xie, Y. Duan, L. Wang, Z. Cheng and J. Cheng, Biosensors and Bioelectronics, 2016, 77, 824-836.

26 C. Petchakup, K. Li and H. Hou, Micromachines, 2017, 8, 87.

27 R.-J. Yang, L.-M. Fu and H.-H. Hou, Sensors and Actuators B: Chemical, 2018, 266, 26-45.

28 A. Vembadi, A. Menachery and M. A. Qasaimeh, Frontiers in Bioengineering and Biotechnology, 2019, 7, 147.

29 E. O. Adekanmbi and S. K. Srivastava, Applied Physics Reviews, 2019, 6, 041313.

30 D. Holmes and H. Morgan, Analytical Chemistry, 2010, 82, 1455-1461.

31 J. S. McGrath, C. Honrado, D. Spencer, B. Horton, H. L. Bridle and H. Morgan, Scientific Reports, 2017, 7, 1-11.

32 P. Ghassemi, X. Ren, B. M. Foster, B. A. Kerr and M. Agah, Biosensors and Bioelectronics, 2020, 150, 111868.

33 S. Gawad, K. Cheung, U. Seger, A. Bertsch and P. Renaud, Lab on a Chip, 2004, 4, 241.

34 D. Spencer and H. Morgan, Lab on a Chip, 2011, 11, 1234.

35 G. Mernier, E. Duqi and P. Renaud, Lab on a Chip, 2012, 12, 4344.

36 T. Zhou, Y. Song, Y. Yuan and D. Li, Analytica Chimica Acta, 2019, 1052, 113-123.

37 J. Cottet, A. Kehren, H. van Lintel, F. Buret, M. Frénéa-Robin and P. Renaud, Microfluidics and Nanofluidics, 2019, 23, 11.

38 X. Xuan, J. Zhu and C. Church, Microfluidics and Nanofluidics, 2010, 9, 1-16.

39 V. Errico, A. D. Ninno, F. R. Bertani, L. Businaro, P. Bisegna and F. Caselli, Sensors and Actuators B: Chemical, 2017, 247, 580-586.

40 F. Caselli, A. De Ninno, R. Reale, L. Businaro and P. Bisegna, Sensors and Actuators B: Chemical, 2018, 256, 580-589.
41 J.-W. Wang, M.-H. Wang and L.-S. Jang, Biosensors and Bioelectronics, 2010, 25, 1271-1276.

42 L. I. Segerink, A. J. Sprenkels, J. G. Bomer, I. Vermes and A. v. d. Berg, Lab on a Chip, 2011, 11, 1995-2001.

43 I. Bilican, M. T. Guler, M. Serhatlioglu, T. Kirindi and C. Elbuken, Sensors and Actuators B: Chemical, 2020, 307, 127531.

44 H. Wang, N. Sobahi and A. Han, Lab on a Chip, 2017, 17, 1264-1269.

45 R. Reale, A. De Ninno, L. Businaro, P. Bisegna and F. Caselli, Microfluidics and Nanofluidics, 2018, 22, 41.

46 M. Solsona, E. Y. Westerbeek, J. G. Bomer, W. Olthuis and A. v. d. Berg, Lab on a Chip, 2019, 19, 1054-1059.

47 H. Fricke, Physical Review, 1925, 26, 678.

48 H. Fricke, The Journal of Physical Chemistry, 1955, 59, 168170.

49 H. Pauly and H. P. Schwan, Zeitschrift für Naturforschung B, 1959, 14, 125-131.

50 K. Asami, T. Hanai and N. Koizumi, Japanese Journal of Applied Physics, 1980, 19, 359.

51 P. R. C. Gascoyne, F. F. Becker and X. B. Wang, Bioelectrochemistry and Bioenergetics, 1995, 36, 115-125.

52 T. Kakutani, S. Shibatani and M. Sugai, Bioelectrochemistry and bioenergetics, 1993, 31, 131-145.

53 J. C. Maxwell, A Treatise on Electricity and Magnetism, Oxford: Clarendon Press, 1873.

54 V. A. Markel, JOSA A, 2016, 33, 1244-1256.

55 A. H. Sihvola and J. A. Kong, IEEE Transactions on Geoscience and Remote Sensing, 1988, 26, 420-429.

56 D. Spencer and H. Morgan, ACS Sensors, 2020, 5, 423-430.

57 T. Hanai, Electrical properties of emulsions, Academic Press, 1968.

58 T. Hanai, T. Imakita and N. Koizumi, Colloid and Polymer Science, 1982, 260, 1029-1034.

59 J. Cottet, O. Fabregue, C. Berger, F. Buret, P. Renaud and M. Frénéa-Robin, Biophysical Journal, 2019, 116, 12-18.

60 A. Irimajiri, T. Suzaki, K. Asami and T. Hanai, Bulletin of the Institute for Chemical Research, KyotoUniversity, 1991, 69, 421-438.

61 A. Mansoorifar, A. Ghosh, A. C. Sabuncu and A. Beskok, IET Nanobiotechnology, 2017, 11, 874-882.

62 R. Liu, W. Waheed, N. Wang, O. Civelekoglu, M. Boya, C.-H. Chu and A. F. Sarioglu, Lab on a Chip, 2017, 17, 2650-2666.

63 P. Jacobs, A. Varlan and W. Sansen, Medical and Biological Engineering and Computing, 1995, 33, 802-810.

64 P. Linderholm and P. Renaud, Lab on a Chip, 2005, 5, 14161417.

65 K. R. Foster and H. P. Schwan, Critical Reviews in Biomedical Engineering, 1989, 17, 25-104.

66 Z. R. Gagnon, ELECTROPHORESIS, 2011, 32, $2466-2487$.

67 T. Sun, D. Holmes, S. Gawad, N. G. Green and H. Morgan, Lab on a Chip, 2007, 7, 1034.

68 A. Valero, T. Braschler and P. Renaud, Lab on a Chip, 2010, 
$10,2216$.

69 B. Hirschorn, M. E. Orazem, B. Tribollet, V. Vivier, I. Frateur and M. Musiani, Electrochimica Acta, 2010, 55, 6218-6227.

70 M. E. Orazem, I. Frateur, B. Tribollet, V. Vivier, S. Marcelin, N. Pébère, A. L. Bunge, E. A. White, D. P. Riemer and M. Musiani, Journal of The Electrochemical Society, 2013, 160, C215.

71 C. A. E. Little, N. D. Orloff, I. E. Hanemann, C. J. Long, V. M. Bright and J. C. Booth, Lab on a Chip, 2017, 17, 2674-2681.

72 J. Riordon, N. M.-Catafard and M. Godin, Applied Physics Letters, 2012, 101, 154105.

73 T. Sun, N. G. Green and H. Morgan, Nano, 2008, 3, 55-63.

74 D. Isèbe and P. Nérin, International Journal for Numerical Methods in Biomedical Engineering, 2013, 29, 462-475.

75 P. Taraconat, J.-P. Gineys, D. Isebe, F. Nicoud and S. Mendez, International Journal for Numerical Methods in Biomedical Engineering, 2019, 35, e3243.

76 T. Sun, N. Green, S. Gawad and H. Morgan, IET Nanobiotechnology, 2007, 1, 69.

77 T. Sun, H. Morgan and N. G. Green, Physical Review E, 2007, 76, 046610.

78 R. Verfürth, Computer Methods in Applied Mechanics and Engineering, 1999, 176, 419-440.

79 H. Pauly and H. P. Schwan, Biophysical Journal, 1966, 6, 621-639.

80 C. Honrado, L. Ciuffreda, D. Spencer, L. Ranford-Cartwright and H. Morgan, Journal of The Royal Society Interface, 2018, 15, 20180416.

81 D. Holmes, D. Pettigrew, C. H. Reccius, J. D. Gwyer, C. v. Berkel, J. Holloway, D. E. Davies and H. Morgan, Lab on a Chip, 2009, 9, 2881-2889.

82 N. Haandbæk, S. C. Bürgel, F. Heer and A. Hierlemann, Lab on a Chip, 2013, 14, 369-377.

83 N. Haandbæk, S. C. Bürgel, F. Rudolf, F. Heer and A. Hierlemann, ACS Sensors, 2016, 1, 1020-1027.

84 K. Cheung, S. Gawad and P. Renaud, 17th IEEE International Conference on Micro Electro Mechanical Systems. Maastricht MEMS 2004 Technical Digest, 2004, pp. 343-346.

85 Y. Zhao, K. Wang, D. Chen, B. Fan, Y. Xu, Y. Ye, J. Wang, J. Chen and C. Huang, Biosensors and Bioelectronics, 2018, 111, 138-143.

86 X. Ren, P. Ghassemi, J. S. Strobl and M. Agah, Biomicrofluidics, 2019, 13, 044103.

87 J. Riordon, M. Nash, M. Calderini and M. Godin, Microelectronic Engineering, 2014, 118, 35-40.

88 U. Larsen, G. Blankenstein and J. Branebjerg, Proceedings of International Solid State Sensors and Actuators Conference (Transducers '97), 1997, pp. 1319-1322 vol.2.

89 J. H. Nieuwenhuis, F. Kohl, J. Bastemeijer, P. M. Sarro and M. J. Vellekoop, Sensors and Actuators B: Chemical, 2004, 102, 44-50.

90 R. Rodriguez-Trujillo, C. A. Mills, J. Samitier and G. Gomila, Microfluidics and Nanofluidics, 2007, 3, 171-176.

91 R. Rodriguez-Trujillo, O. Castillo-Fernandez, M. Garrido,
M. Arundell, A. Valencia and G. Gomila, Biosensors and Bioelectronics, 2008, 24, 290-296.

92 Z. Liu, J. Li, J. Yang, Y. Song, X. Pan and D. Li, Microfluidics and Nanofluidics, 2016, 21, 4.

93 C. Petchakup, H. M. Tay, W. H. Yeap, R. Dalan, S. C. Wong, K. H. H. Li and H. W. Hou, Biosensors and Bioelectronics, 2018, 118, 195-203.

94 J.-C. Chien, A. Ameri, E.-C. Yeh, A. N. Killilea, M. Anwar and A. M. Niknejad, Lab on a Chip, 2018, 18, 2065-2076.

95 M. A. Daniele, D. A. Boyd, D. R. Mott and F. S. Ligler, Biosensors and Bioelectronics, 2015, 67, 25-34.

96 N. Watkins, B. M. Venkatesan, M. Toner, W. Rodriguez and R. Bashir, Lab on a Chip, 2009, 9, 3177-3184.

97 C. Bernabini, D. Holmes and H. Morgan, Lab Chip, 2011, 11, 407-412.

98 H. Choi, C. S. Jeon, I. Hwang, J. Ko, S. Lee, J. Choo, J.-H. Boo, H. C. Kim and T. D. Chung, Lab on a Chip, 2014, 14, 2327-2333.

99 R. Scott, P. Sethu and C. K. Harnett, Review of Scientific Instruments, 2008, 79, 046104.

100 Y. Zhao, Q. Li and X. Hu, Analytical Methods, 2018, 10, 3489-3497.

101 D. Di Carlo, Lab on a Chip, 2009, 9, 3038-3046.

102 J. Zhou and I. Papautsky, Lab on a Chip, 2013, 13, 11211132.

103 D. Di Carlo, D. Irimia, R. G. Tompkins and M. Toner, Proceedings of the National Academy of Sciences, 2007, 104, 1889218897.

104 J. Kim, J. Lee, C. Wu, S. Nam, D. D. Carlo and W. Lee, Lab on a Chip, 2016, 16, 992-1001.

105 H. W. Hou, C. Petchakup, H. M. Tay, Z. Y. Tam, R. Dalan, D. E. K. Chew, K. H. H. Li and B. O. Boehm, Scientific Reports, 2016, 6, 1-12.

106 W. Tang, D. Tang, Z. Ni, N. Xiang and H. Yi, Analytical Chemistry, 2017, 89, 3154-3161.

107 C. Petchakup, H. M. Tay, K. H. H. Li and H. W. Hou, Lab on a Chip, 2019, 19, 1736-1746.

108 C. Raillon, J. Che, S. Thill, M. Duchamp, B. X. E. Desbiolles, A. Millet, E. Sollier and P. Renaud, Cytometry Part A, 2019, 95, 1085-1095.

109 J. Zhang, S. Yan, D. Yuan, G. Alici, N.-T. Nguyen, M. E. Warkiani and W. Li, Lab on a Chip, 2016, 16, 10-34.

110 Y. Ying and Y. Lin, Scientific Reports, 2019, 9, 1-12.

111 P. Mukherjee, X. Wang, J. Zhou and I. Papautsky, Lab on a Chip, 2018, 19, 147-157.

112 Y. Katsumoto, Y. Hayashi, I. Oshige, S. Omori, N. Kishii, A. Yasuda and K. Asami, Biophysical Journal, 2008, 95, 3043-3047.

113 J. S. McGrath, C. Honrado, J. H. Moore, S. J. Adair, W. B. Varhue, A. Salahi, V. Farmehini, B. J. Goudreau, S. Nagdas, E. M. Blais, T. W. Bauer and N. S. Swami, Analytica Chimica Acta, 2020, 1101, 90-98.

114 H. A. Pohl, Journal of Applied Physics, 1951, 22, 869-871.

115 H. A. Pohl and R. Pethig, Journal of Physics E: Scientific In- 
struments, 1977, 10, 190-193.

116 H. A. Pohl, Dielectrophoresis: the behavior of neutral matter in nonuniform electric fields, Cambridge University Press, Cambridge, 1978.

117 M. P. Hughes, Biomicrofluidics, 2016, 10, 032801.

118 R. Pethig, Journal of The Electrochemical Society, 2017, 164, B3049-B3055.

119 R. E. Fernandez, A. Rohani, V. Farmehini and N. S. Swami, Analytica Chimica Acta, 2017, 966, 11-33.

120 J. Yao, G. Zhu, T. Zhao and M. Takei, ELECTROPHORESIS, 2019, 40, 1166-1177.

121 R. Pethig, Biomicrofluidics, 2010, 4, 022811.

122 X. Zhu, K.-W. Tung and P.-Y. Chiou, Applied Physics Letters, 2017, 111, 143506.

123 H. Morgan, H. Morgan, D. Holmes and N. G. Green, IEE Proceedings - Nanobiotechnology, 2003, 150, 76-81.

124 H. Morgan, D. Holmes and N. G. Green, Current Applied Physics, 2006, 6, 367-370.

125 M. Shaker, L. Colella, F. Caselli, P. Bisegna and P. Renaud, Lab on a Chip, 2014, 14, 2548.

126 N. Demierre, T. Braschler, P. Linderholm, U. Seger, H. v. Lintel and P. Renaud, Lab on a Chip, 2007, 7, 355-365.

127 N. Demierre, T. Braschler, R. Muller and P. Renaud, Sensors and Actuators B: Chemical, 2008, 132, 388-396.

128 R. Reale, A. D. Ninno, L. Businaro, P. Bisegna and F. Caselli, ELECTROPHORESIS, 2019, 40, 1400-1407.

129 C.-H. Lin, G.-B. Lee, L.-M. Fu and B.-H. Hwey, Journal of Microelectromechanical Systems, 2004, 13, 923-932.

130 M. Evander, A. J. Ricco, J. Morser, G. T. A. Kovacs, L. L. K. Leung and L. Giovangrandi, Lab on a Chip, 2013, 13, 722729.

131 E. Rollo, E. Tenaglia, R. Genolet, E. Bianchi, A. Harari, G. Coukos and C. Guiducci, Biosensors and Bioelectronics, 2017, 94, 193-199.

132 J. Yang, Y. Huang, X. Wang, X.-B. Wang, F. F. Becker and P. R. C. Gascoyne, Biophysical Journal, 1999, 76, 33073314.

133 A. De Ninno, V. Errico, F. R. Bertani, L. Businaro, P. Bisegna and F. Caselli, Lab on a Chip, 2017, 17, 1158-1166.

134 A. De Ninno, R. Reale, A. Giovinazzo, F. R. Bertani, L. Businaro, P. Bisegna, C. Matteucci and F. Caselli, Biosensors and Bioelectronics, 2019, 111887.

135 F. Caselli and P. Bisegna, Medical Engineering \& Physics, 2017, 48, 81-89.

136 B. Brazey, J. Cottet, A. Bolopion, H. V. Lintel, P. Renaud and M. Gauthier, Lab on a Chip, 2018, 18, 818-831.

137 D. Spencer, F. Caselli, P. Bisegna and H. Morgan, Lab on a Chip, 2016, 16, 2467-2473.

138 D. Yang and Y. Ai, Lab on a Chip, 2019, 19, 3609-3617.

139 F. Caselli, R. Reale, N. A. Nodargi and P. Bisegna, Micromachines, 2017, 8, 283.

140 C. Honrado, J. S. McGrath, R. Reale, P. Bisegna, N. S. Swami and F. Caselli, Analytical and Bioanalytical Chemistry, 2020, 412, 3835-3845.

141 R. Reale, A. D. Ninno, L. Businaro, P. Bisegna and F. Caselli, Lab on a Chip, 2019, 19, 1818-1827.

142 M. Solsona, W. Olthuis and A. van den Berg, Procedia Engineering, 2016, 168, 1446-1449.

143 U. Hassan and R. Bashir, Lab on a Chip, 2014, 14, 43704381.

144 L. Liu, H. Xu, H. Xiu, N. Xiang and Z. Ni, Analyst, 2020, 145, 5128-5133.

145 F. Caselli, A. De Ninno, R. Reale, L. Businaro and P. Bisegna, IEEE Transactions on Biomedical Engineering, 2020, 1-1.

146 C. Cierpka and C. J. Kähler, Journal of Visualization, 2012, $15,1-31$.

147 M. H. Winer, A. Ahmadi and K. C. Cheung, Lab on a Chip, 2014, 14, 1443-1451.

148 M. Kharboutly and M. Gauthier, 2013 IEEE International Conference on Robotics and Automation, 2013, pp. 14461451.

149 J. Zhang, O. Onaizah, K. Middleton, L. You and E. Diller, IEEE Robotics and Automation Letters, 2017, 2, 835-840.

150 S. Jeon, S. Kim, S. Ha, S. Lee, E. Kim, S. Y. Kim, S. H. Park, J. H. Jeon, S. W. Kim, C. Moon, B. J. Nelson, J.-y. Kim, S.-W. Yu and H. Choi, Science Robotics, 2019, 4, 1-11. 